\title{
Kinetic model for the hard-sphere fluid and solid
}

\author{
Andrés Santos \\ Departamento de Física, Universidad de Extremadura, E-06071 Badajoz, Spain \\ José M. Montanero \\ Departamento de Electrónica e Ingeniería Electromecánica, Universidad de Extremadura, E-06071 Badajoz, Spain \\ James W. Dufty \\ Department of Physics, University of Florida, Gainesville, Florida 32611 \\ J. Javier Brey \\ Física Teórica, Universidad de Sevilla, E-41080 Sevilla, Spain
}

(Received 23 June 1997; revised manuscript received 22 September 1997)

\begin{abstract}
The revised Enskog kinetic theory (RET) for a system of hard spheres provides an exceptionally broad description of mesoscopic and macroscopic dynamics, applicable for both fluid and solid phases, long and short wavelengths, low and high densities, and exact at short times. This great potential is compromised by the difficulty of obtaining solutions outside the domain of linear response. In the present work a simpler kinetic model based on the RET is proposed with the same scope of application but accessible to solution for more general states. As a test of this model the rheological properties of a fluid under shear far from equilibrium are calculated as a function of the density and shear rate. Comparisons with Monte Carlo simulations of the RET show excellent agreement for transport properties but some limitations of the model for predicting velocity distributions at large shear rates. The model is discussed critically and its potential for several future applications is noted. [S1063-651X(98)01902-3]

PACS number(s): 47.50. $+\mathrm{d}$, 05.20.Dd, 05.60. $+\mathrm{w}$, 83.50.Ax
\end{abstract}

\section{INTRODUCTION}

Kinetic theory plays a special role as a description of many-body systems intermediate between the detailed underlying microdynamics and phenomenological macroscopic descriptions. For example, the nonlinear Boltzmann kinetic equation provides the basis for calculating a wide range of physical properties for low density gases in states both near and far from equilibrium. The problem of implementing a kinetic theory description is twofold. First, the derivation of the kinetic equation for the one-particle reduced distribution function requires a detailed many-body analysis and a critical assessment of the approximations made, in order to understand the context of its application. In the case of the Boltzmann equation, there is a small parameter, the reduced density, and the kinetic equation is understood to apply at low density and on space and time scales large compared to the force range and collision time, respectively. A corresponding derivation of a kinetic equation at higher densities for general interatomic potentials is still lacking in spite of continual attempts over the past 30 years. The second problem in the application of a given kinetic equation is the technical difficulty encountered in solving it since kinetic equations are nonlinear integro-differential equations for the time dependent distributions in a six dimensional phase space. During the last two decades, this technical problem has been solved for the Boltzmann equation in two ways. One of these is the development of an accurate and efficient Monte Carlo simulation method for constructing the solution [1]. The other method is the use of kinetic model equations obtained as approximate representations of the Boltzmann equation that admit more detailed analytical solution [2]. Together these two methods have provided great new insight into the content of the Boltzmann equation, particularly for states far from equilibrium [3].

Some of the most interesting physical phenomena of current interest lie beyond the validity of the Boltzmann equation. Examples are dense fluid transport far from equilibrium, short wavelength structural dynamics, kinetics of freezing, crystal elasticity and transport, kinetics of metastable and amorphous states. As noted above there is no general kinetic equation available to replace the Boltzmann equation at the fluid and solid densities of interest. A singular exception, however, is the idealized system of hard spheres for which the revised Enskog kinetic theory (RET) provides a remarkably broad description encompassing all of the above phenomena [4]. This kinetic equation is asymptotically exact at short times and therefore has no a priori limitations on the density, space scales, or phase of the states considered. The hard-sphere system supports both fluid and crystal equilibrium states, and the exact equilibrium distributions for these states are stationary solutions to the RET. In addition, there is an $H$ theorem governing the approach to these equilibrium states [5]. For states near equilibrium, the linearized RET has been applied to calculate transport properties and the dynamic structure $S(k, \omega)$ in the fluid phase (using linear kinetic models) [6]. Comparisons with molecular dynamics simulations show good agreement over a wide range of densities and space-time scales. However, its complexity has precluded applications outside the domain of linear response [7]. Recently, the Monte Carlo simulation techniques have been extended to apply to the RET [8], so that 
the full potential of this rich kinetic theory now can be explored. The objective here is to provide the corresponding generalization of the kinetic model method for complementary analytical studies of the full nonlinear RET [9-11,7].

A kinetic model is obtained by replacing the known collision operator with a simpler form that preserves the most important physical properties. These include normalization and the local conservation laws for mass, energy, and momentum. For states near equilibrium, the kinetic equation can be linearized and the construction of a kinetic model for the associated linear collision operator can be made systematic, with any choice being part of a converging sequence of approximations. In the general nonlinear case the construction is more phenomenological. Within the constraints noted above there is a great deal of latitude, and the choice of a particular kinetic model is usually a compromise between simplicity and quantitative accuracy. To illustrate this and to put the proposed model in a broader context, a brief description of kinetic modeling and moment methods is provided in Appendix A. Here we restrict ourselves to a model that reduces in the low density limit to the simplest kinetic model for the Boltzmann equation, the Bhatnagar-Gross-Krook (BGK) model [12]. The BGK model is well tested so that its accuracy and limitations are well known, and the means to correct its limitations are understood. In the present study, we accept such limitations to exploit the simplicity to explore new features of the RET collision operator. These include short wavelength effects due to delocalization of colliding particles, finite density effects, and a stationary state with broken fluid symmetry. The resulting kinetic model provides the same semiquantitative representation for the RET as the BGK model does for the Boltzmann equation. In this respect, it is a significant new tool to explore complex fluid dynamics previously considered prohibitively difficult. As an illustration, we calculate the rheological properties of a dense fluid under shear far from equilibrium and compare them to new Monte Carlo simulations of the RET. The agreement for transport properties (viscosity, viscometric functions, shear dilatancy) is excellent at all densities and shear rates considered, while the velocity distributions show expected discrepancies at the combined conditions of large shear rates and high densities.

In the next section the RET kinetic equation is reviewed. The local conservation laws are derived from it and the finite density "collisional transfer" contributions to the pressure tensor and heat flux are identified. The kinetic model and its relationship to the RET is described in Sec. III. As an initial test of the kinetic model, the Chapman-Enskog solution is obtained for states near equilibrium and the density dependence of the transport coefficients is shown to be the same as that for the RET in the usual first Sonine polynomial approximation. Next, the stationary state of uniform shear flow is defined and the formal solution to the kinetic model equation is given for arbitrary values of the shear rate. A set of nonlinear transport coefficients for momentum transport are calculated analytically and compared favorably with recent results for the RET as a function of the density. Also, the asymptotic behavior of the shear viscosity for large shear rates is obtained showing a surprising transition from shear thinning to shear thickening due to the collisional transfer contributions. The full shear rate dependence of the shear viscosity is calculated and compared with results using the new Monte Carlo simulation method for the RET. The good agreement provides support for both the Monte Carlo method and the kinetic model as effective tools for studying states far from equilibrium at finite densities. The conclusions drawn from these applications of the model are given in the last section and a class of new applications is indicated.

\section{THE REVISED ENSKOG THEORY}

The revised Enskog kinetic equation was derived by van Beijeren and Ernst using a formal many-body analysis of the microdynamics for a system of hard spheres [4]. It is a modification of an earlier phenomenological theory due to Enskog as an attempt to extend the Boltzmann equation to higher densities. The modifications obtained from the formal analysis are essential for a proper treatment of mixtures and to include crystal or metastable states, via a direct connection to density functional theory. The RET gives an equation for the one-particle distribution function, $f(\mathbf{r}, \mathbf{v}, t)$,

$$
\left(\frac{\partial}{\partial t}+\mathbf{v}_{1} \cdot \nabla_{1}\right) f\left(\mathbf{r}_{1}, \mathbf{v}_{1}, t\right)=J_{E}\left[\mathbf{r}_{1}, \mathbf{v}_{1} \mid f(t)\right]
$$

where $J_{E}$ is the Enskog collision operator,

$$
\begin{aligned}
J_{E}\left[\mathbf{r}_{1}, \mathbf{v}_{1} \mid f(t)\right]= & \sigma^{2} \int d \mathbf{v}_{2} \int d \hat{\boldsymbol{\sigma}} \Theta(\hat{\boldsymbol{\sigma}} \cdot \mathbf{g})(\hat{\boldsymbol{\sigma}} \cdot \mathbf{g}) \\
& \times\left\{\chi\left[\mathbf{r}_{1}, \mathbf{r}_{1}-\boldsymbol{\sigma} \mid n(t)\right] f\left(\mathbf{r}_{1}, \mathbf{v}_{1}^{\prime}, t\right)\right. \\
& \times f\left(\mathbf{r}_{1}-\boldsymbol{\sigma}, \mathbf{v}_{2}^{\prime}, t\right)-\chi\left[\mathbf{r}_{1}, \mathbf{r}_{1}+\boldsymbol{\sigma} \mid n(t)\right] \\
& \left.\times f\left(\mathbf{r}_{1}, \mathbf{v}_{1}, t\right) f\left(\mathbf{r}_{1}+\boldsymbol{\sigma}, \mathbf{v}_{2}, t\right)\right\}
\end{aligned}
$$

In the above expression, $\sigma$ is the hard-sphere diameter, $\boldsymbol{\sigma}=\sigma \boldsymbol{\sigma}, \hat{\boldsymbol{\sigma}}$ being a unit vector, $\Theta$ is the Heaviside function, and $\mathbf{g}=\mathbf{v}_{1}-\mathbf{v}_{2}$. The primes on the velocities denote scattered values determined from $\mathbf{v}_{1}^{\prime}=\mathbf{v}_{1}-(\hat{\boldsymbol{\sigma}} \cdot \mathbf{g}) \hat{\boldsymbol{\sigma}}$ and $\mathbf{v}_{2}^{\prime}$ $=\mathbf{v}_{2}+(\hat{\boldsymbol{\sigma}} \cdot \mathbf{g}) \hat{\boldsymbol{\sigma}}$. The differences in the spatial arguments on the right side represent the fact that a colliding pair of particles have their centers separated by $\pm \boldsymbol{\sigma}$. Finally, $\chi\left[\mathbf{r}_{1}, \mathbf{r}_{2} \mid n(t)\right]$ is the pair correlation function for an equilibrium system with nonuniform density field $n(\mathbf{r}, t)$. To be more explicit, $\chi\left[\mathbf{r}_{1}, \mathbf{r}_{2} \mid n(t)\right]$ gives the probability to find particles at $\mathbf{r}_{1}$ and $\mathbf{r}_{2}$ for a system at equilibrium in an external potential $U_{\text {ext }}(\mathbf{r})$. According to a theorem of density functional theory, there is a one to one correspondence between the external potential and the density field for this fictitious equilibrium state [13]. Here, the external potential is that associated with the density field of the actual nonequilibrium state

$$
n(\mathbf{r}, t)=\int d \mathbf{v} f(\mathbf{r}, \mathbf{v}, t)
$$

Thus $\chi[n]$ is an equilibrium functional that can be determined exactly from the second functional derivative of the equilibrium free energy functional for an inhomogeneous state, but it is evaluated at the nonequilibrium density. This 
implies that $\chi[n]$ and, consequently, $J_{E}[f]$ are highly nonlinear functionals of $f$ through this density dependence and Eq. (2.3).

One important property of the dependence of the RET on $\chi[n]$ and the delocalization of the colliding pair, neither effect present in the Boltzmann equation, is the existence of stationary solutions corresponding to equilibrium states of both fluid and crystal phases. They are of the form

$$
f_{s}(\mathbf{r}, \mathbf{v})=n_{s}(\mathbf{r})\left(\frac{\beta m}{2 \pi}\right)^{3 / 2} e^{-\beta m v^{2} / 2}
$$

where $m$ is the mass of a particle, $\beta$ is the temperature parameter, and $n_{s}$ is determined from Eq. (2.1), which leads to [14]

$$
\begin{aligned}
\nabla \ln n_{s}(\mathbf{r}) & =-\sigma^{2} \int d \hat{\boldsymbol{\sigma}} \hat{\boldsymbol{\sigma}} \chi\left[\mathbf{r}, \mathbf{r}+\boldsymbol{\sigma} \mid n_{s}\right] n_{s}(\mathbf{r}+\boldsymbol{\sigma}) \\
& =-\nabla c_{s}^{(1)}\left[\mathbf{r} \mid n_{s}\right] .
\end{aligned}
$$

The first equality can be recognized as the exact first Bogoliubov-Born-Green-Kirkwood-Yvon (BBGKY) hierarchy equation for a hard-sphere stationary state. The second equality recognizes the integral on the right side as the gradient of the one-particle direct correlation function. This is a familiar equation of density functional theory for freezing or melting [13]. For the equilibrium fluid phase, $\chi\left[\mathbf{r}, \mathbf{r}+\boldsymbol{\sigma} \mid n_{s}\right]$ does not depend either on $\mathbf{r}$ or on $\hat{\boldsymbol{\sigma}}$ and the solution of Eq. (2.5) is $n_{s}=$ const. At sufficiently high average density the inhomogeneous density distribution representing the crystal phase also is a nontrivial broken symmetry solution representing lower free energy.

The linearization of Eq. (2.1) around the uniform fluid equilibrium state leads to a kinetic equation that has been studied extensively. Further approximations, equivalent to constructing linear kinetic models, allow calculation of time correlation functions with results that agree favorably with both computer simulation and experiments $[6,15]$. It is significant that this agreement extends over a very wide range of wavelengths (from hydrodynamic to those smaller than the hard-sphere diameter), time scales (from macroscopic to collision times), and densities $\left(0 \leqslant n^{*} \equiv n \sigma^{3}<0.7\right)$. More recently, linearization around the nonuniform crystal equilibrium state also has been studied $[14,16]$. In this way, both linear hydrodynamic equations for states near the fluid equilibrium and linear elasticity equations for crystal states can be derived, with detailed expressions for the transport, elastic, and other coefficients occurring in these equations.

For a general nonequilibrium state, the conservation equations for mass, momentum, and energy are derived from Eq. (2.1) by multiplying with $1, m \mathbf{v}_{1}$, and $\frac{1}{2} m v_{1}^{2}$ and integrating over $\mathbf{v}_{1}$,

$$
\begin{gathered}
\frac{\partial n}{\partial t}+\nabla \cdot(n \mathbf{u})=0, \\
\frac{\partial \mathbf{u}}{\partial t}+\mathbf{u} \cdot \nabla \mathbf{u}+(m n)^{-1} \nabla \cdot \mathrm{P}=0,
\end{gathered}
$$

$$
\frac{\partial e}{\partial t}+\nabla \cdot(e \mathbf{u})+\mathrm{P}: \nabla \mathbf{u}+\nabla \cdot \mathbf{q}=0 .
$$

The flow velocity $\mathbf{u}(\mathbf{r}, t)$ and the internal energy density $e(\mathbf{r}, t)$ are defined by

$$
\begin{gathered}
n(\mathbf{r}, t) \mathbf{u}(\mathbf{r}, t)=\int d \mathbf{v v} f(\mathbf{r}, \mathbf{v}, t), \\
e(\mathbf{r}, t)=\int d \mathbf{v} \frac{m}{2}(\mathbf{v}-\mathbf{u})^{2} f(\mathbf{r}, \mathbf{v}, t) .
\end{gathered}
$$

The pressure tensor $\mathrm{P}(\mathbf{r}, t)$ and the heat flux $\mathbf{q}(\mathbf{r}, t)$ have both "kinetic" and "collisional transfer" contributions, i.e., $\mathrm{P}$ $=\mathrm{P}^{k}+\mathrm{P}^{c}$ and $\mathbf{q}=\mathbf{q}^{k}+\mathbf{q}^{c}$. The kinetic contributions are given by

$$
\begin{aligned}
\mathrm{P}^{k}(\mathbf{r}, t) & =\int d \mathbf{v} m(\mathbf{v}-\mathbf{u})(\mathbf{v}-\mathbf{u}) f(\mathbf{r}, \mathbf{v}, t), \\
\mathbf{q}^{k}(\mathbf{r}, t) & =\int d \mathbf{v} \frac{m}{2}(\mathbf{v}-\mathbf{u})^{2}(\mathbf{v}-\mathbf{u}) f(\mathbf{r}, \mathbf{v}, t),
\end{aligned}
$$

while the divergences of the collisional transfer parts are

$$
\begin{aligned}
\nabla \cdot \mathrm{P}^{c}(\mathbf{r}, t)= & -\int d \mathbf{v} m(\mathbf{v}-\mathbf{u}) J_{E}[\mathbf{r}, \mathbf{v} \mid f(t)], \\
\nabla \cdot \mathbf{q}^{c}(\mathbf{r}, t)= & -\int d \mathbf{v} \frac{m}{2}(\mathbf{v}-\mathbf{u})^{2} J_{E}[\mathbf{r}, \mathbf{v} \mid f(t)] \\
& -\mathrm{P}^{c}(\mathbf{r}, t): \nabla \mathbf{u}(\mathbf{r}, t) .
\end{aligned}
$$

From Eqs. (2.13) and (2.14), the following explicit expressions can be identified:

$$
\begin{aligned}
\mathrm{P}^{c}(\mathbf{r}, t)= & \frac{m}{2} \sigma^{3} \int d \mathbf{v}_{1} \int d \mathbf{v}_{2} \int d \hat{\boldsymbol{\sigma} \Theta}(\hat{\boldsymbol{\sigma}} \cdot \mathbf{g}) \\
& \times(\hat{\boldsymbol{\sigma}} \cdot \mathbf{g})^{2} \hat{\boldsymbol{\sigma}} \hat{\boldsymbol{\sigma}} \int_{0}^{1} d \lambda \chi[\mathbf{r}-(1-\lambda) \boldsymbol{\sigma}, \mathbf{r} \\
& +\lambda \boldsymbol{\sigma} \mid n(t)] f\left(\mathbf{r}-(1-\lambda) \boldsymbol{\sigma}, \mathbf{v}_{1}, t\right) f\left(\mathbf{r}+\lambda \boldsymbol{\sigma}, \mathbf{v}_{2}, t\right), \\
\mathbf{q}^{c}(\mathbf{r}, t)= & \frac{m}{2} \sigma^{3} \int d \mathbf{v}_{1} \int d \mathbf{v}_{2} \int d \hat{\boldsymbol{\sigma}} \Theta(\hat{\boldsymbol{\sigma}} \cdot \mathbf{g}) \\
& \times(\hat{\boldsymbol{\sigma}} \cdot \mathbf{g})^{2}(\widetilde{\mathbf{G}} \cdot \hat{\boldsymbol{\sigma}}) \hat{\boldsymbol{\sigma}} \int_{0}^{1} d \lambda \chi[\mathbf{r}-(1-\lambda) \boldsymbol{\sigma}, \mathbf{r} \\
& +\lambda \boldsymbol{\sigma} \mid n(t)] f\left(\mathbf{r}-(1-\lambda) \boldsymbol{\sigma}, \mathbf{v}_{1}, t\right) f\left(\mathbf{r}+\lambda \boldsymbol{\sigma}, \mathbf{v}_{2}, t\right) .
\end{aligned}
$$

Here, $\widetilde{\mathbf{G}}=\frac{1}{2}\left(\mathbf{v}_{1}+\mathbf{v}_{2}\right)-\mathbf{u}$. The derivation of Eqs. (2.15) and (2.16) is given in Appendix B. The collisional transfer contributions are due to the delocalization of the colliding pair and the additional density dependence of the RET. They vanish in the low density limit but dominate at high densities. These expressions give the collisional transfer parts of the fluxes as explicit velocity independent functionals of $f(\mathbf{r}, \mathbf{v}, t)$ 
and characterize the primary density dependence in the macroscopic conservation laws beyond that which follows from the Boltzmann equation.

\section{KINETIC MODEL FOR THE RET}

Models for linear kinetic equations can be constructed by approximations to the spectral decomposition of the linear collision operator. In the case of nonlinear kinetic equations there is less guidance and greater flexibility, depending on the desired properties to preserve. This is illustrated by a brief discussion of kinetic models and moment methods in Appendix A. We construct a kinetic model with the same qualitative features as the Enskog equation by first identifying the essential features of the collision operator $J_{E}[f]$ necessary for the conservation laws. It follows from the brief discussion of the preceding section that the kinetic parts of the fluxes arise from the free streaming term $\mathbf{v} \cdot \nabla f$ rather than from the collision operator. The remaining collisional transfer parts of the fluxes come from moments of the collision operator with respect to $\left\{1, \mathbf{v}, v^{2}\right\}$, so these moments must be preserved exactly by any approximate kinetic model for the collision operator. To extract this feature, the variables $\mathbf{r}$ and $t$ are fixed and $J_{E}[f]$ is considered as a function of the velocity. It is convenient to introduce a Hilbert space defined by the scalar product

$$
(\xi, \zeta)=\int d \mathbf{v} \phi \ell(\mathbf{v}) \xi^{*}(\mathbf{v}) \zeta(\mathbf{v})
$$

where $\phi_{\ell}(\mathbf{v})$ is related to the local equilibrium distribution $f_{\ell}(\mathbf{v})$ by

$$
f_{\ell}(\mathbf{v})=n \phi_{\curlywedge}(\mathbf{v}), \quad \phi_{\curlywedge}(\mathbf{v})=\left(\frac{\beta m}{2 \pi}\right)^{3 / 2} \exp \left(-\beta m V^{2} / 2\right) .
$$

Here we have introduced the peculiar velocity $\mathbf{V}=\mathbf{v}-\mathbf{u}$ and $\beta=\left(k_{B} T\right)^{-1}$, where $k_{B}$ is the Boltzmann constant and $T$ is the nonequilibrium temperature defined by $e=\frac{3}{2} n k_{B} T$. The functions $\left\{1, \mathbf{v}, v^{2}\right\}$ span a subspace in this Hilbert space and an orthonormal basis for this subspace is given by

$$
\left\{\psi_{\alpha}\right\}=\left\{1,(m \beta)^{1 / 2} \mathbf{V},\left(\frac{2}{3}\right)^{1 / 2}\left(\frac{m \beta}{2} V^{2}-\frac{3}{2}\right)\right\} .
$$

Finally, a projection operator $\mathcal{P}$ onto this set is given by

$$
\mathcal{P} g(\mathbf{v})=\sum_{\alpha} \psi_{\alpha}(\mathbf{v}) \phi_{\ell}(\mathbf{v})\left(\psi_{\alpha}, \phi_{\ell}^{-1} g\right)
$$

With these definitions, the RET collision operator can be decomposed into the two parts

$$
J_{E}[f]=\mathcal{P} J_{E}[f]+(1-\mathcal{P}) J_{E}[f] .
$$

The first term on the right hand side gives the collisional transfer contributions to the fluxes in the conservation equations, and must be retained in any acceptable kinetic model. Since the second term on the right side of Eq. (3.5) does not contribute to the form of the conservation laws, the simplest approximation is to represent $J_{E}[f]$ in this "less important", subspace as the negative of an effective collision frequency $\lambda$ times the distribution function [9],

$$
J_{E}[f] \rightarrow \mathcal{P} J_{E}[f]-(1-\mathcal{P}) \lambda f
$$

This choice has all the qualitative features discussed for the RET, regardless of the choice for $\lambda$, and is the primary form of the kinetic model we propose.

In Ref. [9] $\lambda$ was chosen to be a velocity independent functional of $f$ through a dependence on the local density and temperature. Here we consider a generalization to include a possible velocity dependence

$$
\lambda[\mathbf{v} \mid f]=\nu[f]+\delta \nu[\mathbf{v} \mid f],
$$

where $\nu$ is independent of the velocity. To further constrain the form of $\lambda$, let us particularize the model collision operator to the local equilibrium state:

$$
J_{E}\left[f_{\ell}\right] \rightarrow J_{E}\left[f_{\ell}\right]-(1-\mathcal{P})\left\{\delta \nu(\mathbf{v}) f_{\ell}+J_{E}\left[f_{\ell}\right]\right\},
$$

where use has been made of the property $(1-\mathcal{P}) \nu f_{\ell}$ $=\nu(1-\mathcal{P}) f_{\ell}=0$. Equation (3.8) suggests the choice

$$
\delta \nu(\mathbf{v})=-f^{-1} J_{E}\left[f_{\ell}\right] .
$$

The interpretation of $\lambda f$ is the change of $f$ due to collisions that affect only the component $(1-\mathcal{P}) f$. There are two parts. The first represents an average collision rate $\nu$ depending on the local temperature and density. The second represents an additional collision rate due to spatial inhomogeneities of the local equilibrium state (recall $J_{E}\left[f_{\ell}\right]$ vanishes for the homogeneous equilibrium state). The latter is a collisional transfer effect associated with the difference in position of the colliding particles.

The inclusion of $\delta \nu(\mathbf{v})$ leads to a quantitative improvement of the predicted transport coefficients relative to the model of Ref. [9] with $\delta \nu(\mathbf{v})=0$, but otherwise provides no new qualitative change. However, the price of this quantitative improvement is an additional complex velocity dependence, beyond the simple polynomial dependence of $\mathcal{P} J_{E}[f]$. This undesired complication of the kinetic model can be eliminated by retaining only the lowest order polynomial dependence of $(1-\mathcal{P}) \delta \nu f$. Closer inspection shows that the Chapman-Enskog solution (to first order in the gradients) and transport coefficients depend only on moments of $J_{E}\left[f_{\ell}\right]$ with respect to two functions in the subspace of $(1-\mathcal{P})$,

$$
\mathrm{D}(\mathbf{V})=m\left(\mathbf{V} \mathbf{V}-\frac{1}{3} V^{2} \mathbb{1}\right), \quad \mathbf{S}(\mathbf{V})=\left(\frac{m}{2} V^{2}-\frac{5}{2 \beta}\right) \mathbf{V}
$$

1 being the unit tensor. Retaining only the projections of $\delta \nu f$ along these two functions gives

$$
\lambda \rightarrow \nu-\left(f_{\ell} / f\right) \beta[\mathrm{A}: \mathrm{D}(\mathbf{V})+\mathbf{B} \cdot \mathbf{S}(\mathbf{V})],
$$

where

$$
\mathrm{A}=\frac{\beta}{2 n} \int d \mathbf{V D}(\mathbf{V}) J_{E}\left[f_{\ell}\right], \quad \mathbf{B}=\frac{2 m \beta^{2}}{5 n} \int d \mathbf{V S}(\mathbf{V}) J_{E}\left[f_{\ell}\right] .
$$


The model kinetic equation is obtained by substituting Eq. (3.11) into Eqs. (3.6) and (2.1). The contribution from $\mathcal{P} J_{E}[f]$ in Eq. (3.11) can be made more explicit in terms of the collisional transfer contributions to the fluxes, $\mathrm{P}^{c}$ and $\mathbf{q}^{c}$, using Eqs. (2.13) and (2.14). The resulting kinetic equation is

$$
\begin{aligned}
\left(\frac{\partial}{\partial t}+\mathbf{v} \cdot \nabla\right) f= & -\nu\left(f-f_{\ell}\right)-\frac{\beta}{n} f_{\ell}\left[\mathbf{V} \nabla: \mathrm{P}^{c}+\left(\frac{m \beta}{3} V^{2}-1\right)\right. \\
& \left.\times\left(\nabla \cdot \mathbf{q}^{c}+\mathrm{P}^{c}: \nabla \mathbf{u}\right)\right] \\
& +\beta f \_[\mathrm{A}: \mathrm{D}(\mathbf{V})+\mathbf{B} \cdot \mathbf{S}(\mathbf{V})]
\end{aligned}
$$

The macroscopic conservation laws follow from this equation, by construction, and it is straightforward to confirm that the stationary solutions obey Eq. (2.5) with the exact fluid and crystal equilibrium solutions.

Equation (3.13) is the kinetic model considered in the remainder of this paper. It is not self-evident in what respect this model equation is more tractable than the underlying RET kinetic equation. Structurally, Eq. (3.13) is still a highly nonlinear integro-differential equation. The parameters of $f_{\ell}$ are functionals of $f$ through their definitions (2.3), (2.9), and (2.10). Similarly, $\mathrm{P}^{c}$ and $\mathbf{q}^{c}$ are functionals of $f$ both explicitly and implicitly through the density dependence of $\chi$. The essential advantage of Eq. (3.13) over the RET is that these functionals are independent of the velocity, and that the velocity dependence of the model collision operator on the right side of Eq. (3.13) is simple: Gaussians times polynomials of degree 3 . Consequently, for given initial and boundary conditions, an implicit solution to the kinetic equation can be obtained displaying the exact velocity dependence but parametrized by these space dependent functionals. Use of this implicit solution in the definitions of the functionals provides velocity independent integral equations for the functionals and hence the full solution. In this way the original problem in six dimensional phase space is reduced to one in three dimensional coordinate space. The problem remains quite difficult in general, but becomes tractable in many cases where additional simplifications such as symmetries can be exploited. A nontrivial example of this method for solving the model kinetic equation is given in Sec. V.

\section{CHAPMAN-ENSKOG SOLUTION}

For states near equilibrium or near spatial uniformity, the kinetic equation can be solved approximately by expansion in a small parameter. In the first case the deviation from the stationary solution, $\delta f=f-f_{s}$, is the small parameter and to leading order the kinetic equation can be linearized. The linear kinetic equation then can be solved exactly (reduced to quadratures) for the full spectrum of excitations and for all space and time scales. The result is quite close to existing kinetic models for the linearized RET and will not be discussed here. A different approach is based on the local stationary distribution $f_{\ell}$ as the reference state and the construction of a solution whose space and time dependence occurs entirely through the conserved local densities, $n(\mathbf{r}, t)$, $\mathbf{u}(\mathbf{r}, t)$, and $e(\mathbf{r}, t)$. Such a solution is called "normal" and is appropriate for a derivation of closed hydrodynamic equations from the conservation equations (2.6)-(2.8). The
Chapman-Enskog method [17] yields such a solution for states near spatial uniformity as an expansion in gradients of the local densities. In this section we restrict attention to the fluid phase and apply the Chapman-Enskog method to derive the solution to first order in the gradients. This solution then is used to calculate the pressure tensor and heat flux so that the pressure, shear viscosity, bulk viscosity, and thermal conductivity can be identified as functions of the density.

The distribution function $f$ is expressed as the local equilibrium contribution plus a term of first order in the gradients of the conserved densities, $f=f_{\ell}+\epsilon f^{(1)}+\cdots$, where $\epsilon$ is a formal uniformity parameter introduced to order the gradient expansion and set equal to one in the final results. Use of this expansion in the definitions for the fluxes gives a corresponding expansion for these quantities, e.g., $P=P^{(0)}$ $+\epsilon \mathrm{P}^{(1)}+\cdots$. Similarly, use of these fluxes in the local conservation equations leads to an identification of the time derivatives of the fields as an expansion in the gradients, $\partial_{t}$ $=\partial_{t}^{(0)}+\epsilon \partial_{t}^{(1)}+\cdots$. Consider first the conservation laws to zeroth order in $\epsilon$. Equations (2.6)-(2.8) give $\partial_{t}^{(0)}=0$. Accordingly, the kinetic model equation (3.13) becomes

$$
0=\mathrm{A}^{(0)}: \mathrm{D}(\mathbf{V})+\mathbf{B}^{(0)} \cdot \mathbf{S}(\mathbf{V}) \text {. }
$$

For consistency, therefore, we must have $A^{(0)}=\mathbf{B}^{(0)}=0$. This can be verified by direct calculation from the definitions (3.12), which shows that $\mathbf{A}$ and $\mathbf{B}$ are of first order in the gradients

$$
\mathrm{A}=-\epsilon \frac{2}{15} \pi n^{*} \chi_{e} \widetilde{\nabla \mathbf{u}}+\cdots, \quad \mathbf{B}=-\epsilon \frac{2}{5} \pi n^{*} \chi_{e} \nabla \ln T+\cdots,
$$

where $\chi_{e}$ is the pair correlation function at contact for a homogeneous fluid, $n^{*}=n \sigma^{3}$, and $2 \widetilde{\nabla \mathbf{u}}$ denotes the symmetric, traceless part of the tensor $\nabla \mathbf{u}$. To this order, the heat flux vanishes from symmetry and the pressure tensor is given in terms of the kinetic and collisional transfer contributions to the hydrostatic pressure

$$
\mathrm{P}^{(0)}=\left(p^{k}+p^{c}\right) 1=p \rrbracket, \quad p=n \beta^{-1}\left(1+\frac{2}{3} \pi n^{*} \chi_{e}\right) .
$$

The last equation gives the exact equation of state for the hard-sphere fluid.

To first order in $\epsilon$, the kinetic equation becomes

$$
\begin{aligned}
\left(\partial_{t}^{(1)}+\mathbf{v} \cdot \nabla\right) f_{\ell}= & -\nu f^{(1)}-\frac{\beta}{n} f_{\ell}\left[\mathbf{V} \cdot \nabla p^{c}\right. \\
& \left.+\left(\frac{m \beta}{3} V^{2}-1\right) p^{c} \nabla \cdot \mathbf{u}\right] \\
& +\beta f\left[\mathrm{~A}^{(1)}: \mathrm{D}(\mathbf{V})+\mathbf{B}^{(1)} \cdot \mathbf{S}(\mathbf{V})\right] .
\end{aligned}
$$

The first order time derivatives from Eqs. (2.6)-(2.8) are

$$
\begin{gathered}
\partial_{t}^{(1)} n=-\nabla \cdot(n \mathbf{u}), \\
\partial_{t}^{(1)} \mathbf{u}=-\mathbf{u} \cdot \nabla \mathbf{u}-(m n)^{-1} \nabla p,
\end{gathered}
$$




$$
\partial_{t}^{(1)} e=-\nabla \cdot(e \mathbf{u})-p \nabla \cdot \mathbf{u}
$$

Use of these results in Eq. (4.4) gives the desired result

$$
\begin{aligned}
f^{(1)}= & -\nu^{-1} \beta f_{\ell}\left[\left(1+\frac{4 \pi}{15} n^{*} \chi_{e}\right) \mathrm{D}(\mathbf{V}): \nabla u\right. \\
& \left.+\left(1+\frac{2 \pi}{5} n^{*} \chi_{e}\right) \mathbf{S}(\mathbf{V}) \cdot \nabla \ln T\right] .
\end{aligned}
$$

This has the same form as is obtained by the RET, except that in that case $D(\mathbf{V})$ and $\mathbf{S}(\mathbf{V})$ are replaced by solutions to linear integral equations. Accurate approximations to the solutions are given by the leading terms of Sonine polynomial expansions, resulting in the same velocity dependence as in Eq. (4.8). This agreement can be enhanced further by choosing the model collision frequency $\nu$ to give the same coefficient for either the $\nabla \mathbf{u}$ term or the $\nabla \ln T$ term. However, it is not possible to obtain agreement of both terms with the RET. This is a shortcoming already present in the BGK kinetic model at low density and of course appears here as well. Still, it is significant that the additional density dependence of each term in Eq. (4.8) is exactly the same as that for the RET.

The pressure tensor and heat flux now can be calculated to first order in the gradients using Eq. (4.8) in Eqs. (2.11)(2.16). The integrations are straightforward but lengthy and similar to those described in Appendix D of Ref. [18]. The results are

$$
\mathrm{P}^{(1)}=-\eta \widetilde{\nabla \mathbf{u}}-\kappa(\nabla \cdot \mathbf{u}) 1, \quad \mathbf{q}^{(1)}=-\lambda \nabla T,
$$

where $\eta$ is the shear viscosity, $\kappa$ is the bulk viscosity, and $\lambda$ is the thermal conductivity,

$$
\begin{gathered}
\eta=\frac{n}{\beta \nu}\left(1+\frac{4 \pi}{15} n^{*} \chi_{e}\right)^{2}+\frac{3}{5} \kappa, \\
\lambda=\frac{5 n k_{B}}{2 m \beta \nu}\left(1+\frac{2 \pi}{5} n^{*} \chi_{e}\right)^{2}+\frac{3 k_{B}}{2 m} \kappa, \\
\kappa=\frac{4}{9} n^{2} \sigma^{4} \chi_{e}(\pi m / \beta)^{1 / 2} .
\end{gathered}
$$

The bulk viscosity is independent of the parameter $\nu$ and is the same as that obtained from the RET. As noted above, $\nu$ may be chosen to assure the correct shear viscosity or the correct thermal conductivity. For example, the correct RET viscosity is given by the choice

$$
\nu=\frac{16}{5} \sqrt{\pi} n \sigma^{2} \chi_{e}(\beta m)^{-1 / 2}
$$

The thermal conductivity for the model for this choice is smaller than that for the RET by a factor between $2 / 3$ and $(6 \pi+32) /(9 \pi+32) \simeq 0.84$. For practical purposes the pair correlation function at contact can be approximated by the Carnahan-Starling form [19]

$$
\chi_{e} \rightarrow\left(1-\frac{\pi}{12} n^{*}\right) /\left(1-\frac{\pi}{6} n^{*}\right)^{3}
$$

\section{APPLICATION TO UNIFORM SHEAR FLOW}

The analysis of the preceding section confirms that the kinetic model represents the RET well for states near equilibrium. However, the greatest potential of the model is for situations far from equilibrium, where analysis of the RET (or even the Boltzmann equation) is prohibitively difficult. As an illustrative example and a more stringent test of the model, we consider here the state of uniform shear flow (USF). This state has been studied extensively by molecular dynamics simulation to analyze rheological properties in simple atomic fluids [20]. The macroscopic state is characterized by spatially uniform temperature and pressure, and a linear flow field: $\mathbf{u}(\mathbf{r})=\mathrm{a} \cdot \mathbf{r}=a y \hat{\mathbf{x}}$, where $\mathrm{a}=a \hat{\mathbf{x}} \hat{\mathbf{y}}$. The constant shear rate $a$ is a single control parameter that can be chosen to drive the system arbitrarily far from equilibrium. The appropriate boundary conditions for USF are those of Lees and Edwards [21], which are simple periodic boundary conditions in the local Lagrangian frame [22]. The resulting shear produces viscous heating that is compensated by an external nonconservative force (thermostat), $\mathbf{F}=-m \alpha(a) \mathbf{V}$, where the thermostat parameter $\alpha(a)$ is defined to assure that the temperature remains constant.

Uniform shear flow is therefore a stationary state with a particularly simple spatial inhomogeneity. Even the latter can be suppressed in the kinetic equation by a transformation to the relative velocity $\mathbf{V}=\mathbf{v}-\mathbf{u}(\mathbf{r})$. The stationary solution then has the form $f(\mathbf{r}, \mathbf{v}, t)=f(\mathbf{V})$ and Eq. (3.13) becomes

$$
\begin{aligned}
& -\left(a V_{y} \frac{\partial}{\partial V_{x}}+\alpha \frac{\partial}{\partial \mathbf{V}} \cdot \mathbf{V}-\nu\right) f \\
& =\beta f \_\left[\beta^{-1} \nu-n^{-1} P_{x y}^{c} a\left(\frac{m \beta}{3} V^{2}-1\right)\right. \\
& +\mathrm{A}: \mathrm{D}(\mathbf{V})+\mathbf{B} \cdot \mathbf{S}(\mathbf{V})] .
\end{aligned}
$$

In these variables there is no longer any space dependence in the problem. The first term on the left hand side represents an inertial force due to the velocity transformation, while the second term is due to the thermostat [23]. On the right hand side we have taken into account that in the USF the heat flux vanishes and the pressure tensor is uniform. Since the complete velocity dependence of the right side is given explicitly, the equation can be solved to determine $f$ in terms of the constants $\mathrm{A}(a), \mathbf{B}(a), \alpha(a)$, and $P_{x y}^{c}(a)$. The first two constants are determined entirely by the local equilibrium distribution specialized to uniform shear flow, according to Eq. (3.12). Since $\mathbf{B}(a)$ is a vector and no vector can be constructed from the tensor a alone, it follows that $\mathbf{B}(a)=0$. The nonvanishing elements of $\mathrm{A}(a)$ are found to be 


$$
\begin{aligned}
A_{x x}= & A_{y y} \\
= & -\frac{1}{2} A_{z z}=(m \beta)^{-1 / 2} n \sigma^{2} \chi_{e} \int d \hat{\boldsymbol{\sigma}}\left(\hat{\sigma}_{x}^{2}-\frac{1}{3}\right) \\
& \times \bar{a} \hat{\sigma}_{x} \hat{\sigma}_{y}\left[\frac{2}{\pi^{1 / 2}} \bar{a} \hat{\sigma}_{x} \hat{\sigma}_{y} e^{-\bar{a}^{2} \hat{\sigma}_{x}^{2} \hat{\sigma}_{y}^{2}}\right. \\
& \left.+\left(1+2 \bar{a}^{2} \hat{\sigma}_{x}^{2} \hat{\sigma}_{y}^{2}\right) \operatorname{erf}\left(\bar{a} \hat{\sigma}_{x} \hat{\sigma}_{y}\right)\right], \\
A_{x y} & =-\frac{4 \pi}{15}(m \beta)^{-1 / 2} n \sigma^{2} \chi_{e} \bar{a}\left(1+\frac{2}{7} \bar{a}^{2}\right),
\end{aligned}
$$

where $\bar{a} \equiv \frac{1}{2} a \sigma(m \beta)^{1 / 2}$ and, for uniform density, $\chi[\mathbf{r}, \mathbf{r}$ $+\boldsymbol{\sigma} \mid n] \rightarrow \chi_{e}(n)$. Next, the constant $\alpha(a)$ characterizing the thermostat is determined by requiring that the temperature be constant. There is an additional term in the energy equation (2.8) due to this external force, $-3 n \alpha(a) / \beta$. For a stationary state this must cancel the viscous heating, which gives the defining equation for $\alpha(a)$ in terms of $P_{x y}(a)$,

$$
\alpha(a)=-\beta a P_{x y}(a) / 3 n .
$$

The kinetic and collisional transfer parts of $P_{x y}(a)$ are explicit functionals of $f$ given by Eqs. (2.11) and (2.15), respectively. Since Eq. (5.1) gives $f$ as a function of $P_{x y}^{c}(a)$, these can be determined self-consistently.

In order to ease the notation, we choose units such that $\nu=1, m=1$, and $m \beta / 2=1$. This implies that the mean free path also equals one and consequently the diameter $\sigma$, expressed as a multiple of the mean free path, is a function of density, $\sigma=\frac{8}{5} \sqrt{2 \pi} n^{*} \chi_{e}(n)$. In these units, Eq. (5.1) can be written as

$$
\begin{gathered}
\mathcal{L} f(\mathbf{V})=\widetilde{f} \iota(\mathbf{V}), \\
\widetilde{f}(\mathbf{V}) \equiv f \curlywedge(\mathbf{V})\left[1-\frac{4}{3}\left(V^{2}-\frac{3}{2}\right) a n^{-1} P_{x y}^{c}+2 \mathrm{~A}: \mathrm{D}(\mathbf{V})\right],
\end{gathered}
$$

where

$$
\mathcal{L}=(1-3 \alpha)-a V_{y} \frac{\partial}{\partial V_{x}}-\alpha \mathbf{V} \cdot \frac{\partial}{\partial \mathbf{V}}
$$

The solution is

$$
\begin{aligned}
f(\mathbf{V}) & \left.=\int_{0}^{\infty} d s e^{-s \mathcal{L}} \widetilde{f} / \mathbf{V}\right) \\
& =\int_{0}^{\infty} d s e^{-(1-3 \alpha) s} \widetilde{f} /\left(e^{\alpha s}(1+s \mathrm{a}) \cdot \mathbf{V}\right),
\end{aligned}
$$

where use has been made of the properties $\exp \left(a t v_{x} \partial_{v_{x}}\right) X\left(v_{x}\right)=X\left(e^{a t} v_{x}\right) \quad$ and $\exp \left(a t v_{y} \partial_{v_{x}}\right) X\left(v_{x}\right)=X\left(v_{x}\right.$ $\left.+a t v_{y}\right)$. From this formal solution, the kinetic part of the pressure tensor $P_{x y}^{k}(a)$ can be calculated directly by multiplication with $m V_{x} V_{y}$ and integration over the velocity to give

$$
\begin{aligned}
P_{x y}^{k}= & -a \frac{n}{2}(1+2 \alpha)^{-2}\left[1-\frac{4}{3} a n^{-1} P_{x y}^{c}+2 A_{x x}\right. \\
& \left.-2 a^{-1}(1+2 \alpha) A_{x y}\right] .
\end{aligned}
$$

Substitution of this into Eq. (5.4) yields a cubic equation for $\alpha(a)$ in terms of $P_{x y}^{c}(a)$,

$$
\begin{aligned}
& 3\left(2 \alpha+\frac{4}{3} a n^{-1} P_{x y}^{c}\right)(1+2 \alpha)^{2}+4(1+2 \alpha) a A_{x y} \\
& =2 a^{2}\left(1-\frac{4}{3} a n^{-1} P_{x y}^{c}+2 A_{x x}\right)
\end{aligned}
$$

which has only one real root. Finally, $P_{x y}^{c}$ is obtained from Eq. (2.15),

$$
\begin{aligned}
P_{x y}^{c}= & \frac{m}{2} \sigma^{3} \chi_{e} \int d \hat{\boldsymbol{\sigma}} \hat{\sigma}_{x} \hat{\sigma}_{y} \int d \mathbf{V}_{1} \int d \mathbf{V}_{2} \Theta(\hat{\boldsymbol{\sigma}} \cdot \mathbf{g})(\hat{\boldsymbol{\sigma}} \cdot \mathbf{g})^{2} \\
& \times f\left(\mathbf{V}_{1}+\mathrm{a} \cdot \boldsymbol{\sigma}\right) f\left(\mathbf{V}_{2}\right)
\end{aligned}
$$

where for uniform shear flow $\mathbf{u}(\mathbf{r}+\lambda \boldsymbol{\sigma})-\mathbf{u}[\mathbf{r}-(1-\lambda) \boldsymbol{\sigma}]$ $=\mathrm{a} \cdot \boldsymbol{\sigma}$, with $\mathrm{a}=a \hat{\mathbf{x}} \hat{\mathbf{y}}$.

Equations (5.7), (5.9), and (5.10) show that the solution to the kinetic equation has been reduced to quadratures. Substitution of Eq. (5.7) into Eq. (5.10) and performing the velocity integrals provides an algebraic equation for $P_{x y}^{c}(a)$ in terms of $\alpha(a)$. Together with Eq. (5.9) these two equations determine the shear rate dependence of both $P_{x y}^{c}(a)$ and $\alpha(a)$. Use of these forms in Eq. (5.7) then completely determines the velocity distribution. All properties of interest can be obtained from integrals over this distribution. In general this self-consistent procedure must be implemented numerically. However, to illustrate the approach and to test the predictive quality of the kinetic model we first extract the analytic results for small and large shear rates.

\section{A. Solution for small shear rates}

Assume a series expansion for the distribution function in powers of $a$ :

$$
f=f_{\ell}+a f^{(1)}+a^{2} f^{(2)}+a^{3} f^{(3)}+\cdots .
$$

This implies

$$
\begin{gathered}
P_{x y}(a)=a P_{x y}^{(1)}+a^{3} P_{x y}^{(3)}+\cdots, \\
\alpha(a)=a^{2} \alpha^{(2)}+a^{4} \alpha^{(4)}+\cdots .
\end{gathered}
$$

The fact that $P_{x y}(a)$ is an odd function of $a$ and $\alpha(a)$ is an even function follows from considerations of symmetry under space inversion of the $x$ or $y$ axes. It follows directly from Eq. (5.9) that

$$
\alpha^{(2)}=\frac{1}{3}\left(1+\frac{4 \pi}{15} n^{*} \chi_{e}-2 n^{-1} P_{x y}^{c(1)}\right) .
$$

Use of Eq. (5.13) in the formal solution (5.7) allows expansion up through $a^{3}$ with the results 


$$
\begin{aligned}
& f^{(1)}(\mathbf{V})=-2\left(1+\frac{4 \pi}{15} n^{*} \chi_{e}\right) V_{x} V_{y} f \curlywedge(\mathbf{V}), \\
& f^{(2)}(\mathbf{V})=\left[\left(1+\frac{4 \pi}{15} n^{*} \chi_{e}\right)\left(1-\frac{2}{3} V^{2}-2 V_{y}^{2}+4 V_{x}^{2} V_{y}^{2}\right)\right. \\
&\left.+\frac{256 \pi}{1575}\left(n^{*} \chi_{e}\right)^{2}\left(V^{2}-3 V_{z}^{2}\right)\right] f_{\ell}(\mathbf{V}), \\
& f^{(3)}(\mathbf{V})= 4 V_{x} V_{y}\left\{( 1 + \frac { 4 \pi } { 1 5 } n ^ { * } \chi _ { e } ) \left[3 V_{y}^{2}-2 V_{x}^{2} V_{y}^{2}\right.\right. \\
&\left.+\frac{2}{3}\left(1-n^{-1} P_{x y}^{c(1)}+\frac{2 \pi}{15} n^{*} \chi_{e}\right)\left(V^{2}-\frac{5}{2}\right)\right] \\
&-\frac{256 \pi^{2}}{2625}\left(n^{*} \chi_{e}\right)^{3}-\frac{128 \pi}{1575}\left(n^{*} \chi_{e}\right)^{2} \\
&\left.\times\left(V^{2}-3 V_{z}^{2}-1\right)\right\} f(\mathbf{V}) .
\end{aligned}
$$

To this order, only $P_{x y}^{c(1)}$ remains undetermined in Eq. (5.16). This is obtained directly by using Eq. (5.14) in the expansion of Eq. (5.10),

$$
P_{x y}^{c(1)}=-n \frac{2 \pi}{15} n^{*} \chi_{e}\left[1+\frac{4}{5}\left(4+\frac{\pi}{3}\right) n^{*} \chi_{e}\right] .
$$

Further details of these calculations are given in Appendix C. Equations (5.14)-(5.17) completely determine the distribution function through order $a^{3}$.

As an illustration of these small shear rate results, the shear viscosity, viscometric functions, and shear dilatancy are calculated. These transport properties characterize the non-Newtonian effects of the shear rate beyond NavierStokes order hydrodynamics. The shear rate dependent shear viscosity is defined as

$$
\eta(a)=-\frac{P_{x y}}{a}
$$

the viscometric functions are defined by

$$
\Psi_{1}(a)=\frac{P_{y y}-P_{x x}}{a^{2}}, \quad \Psi_{2}(a)=\frac{P_{z z}-P_{y y}}{a^{2}},
$$

and the shear dilatancy by

$$
\gamma(a)=\frac{p(a)-p(0)}{a^{2}}, \quad p(a)=\frac{1}{3} \operatorname{TrP}(a) .
$$

These four scalar functions are sufficient to represent the complete pressure tensor $P$. They are calculated from Eqs. (2.11) and (5.10) for the kinetic and collisional transfer contributions to the pressure tensor, using the above expansion for the distribution function. The details are given in Appendix $\mathrm{C}$, leading to the results

$$
\begin{aligned}
\eta(a)= & \eta(0)-\eta_{B} \chi_{e}^{-1}\left[\frac{4}{3}+\frac{16 \pi}{15} n^{*} \chi_{e}+\frac{16 \pi}{225}\left(\frac{41}{7}+\frac{14 \pi}{3}\right)\right. \\
& \times\left(n^{*} \chi_{e}\right)^{2}+\frac{256 \pi^{2}}{3375}\left(1+\frac{2 \pi}{3}\right)\left(n^{*} \chi_{e}\right)^{3}-\frac{512 \pi^{2}}{16875}\left(\frac{232}{49}\right. \\
& \left.\left.+\frac{13 \pi}{42}-\frac{\pi^{2}}{9}\right)\left(n^{*} \chi_{e}\right)^{4}\right] a^{2}+O\left(a^{4}\right),
\end{aligned}
$$

where $\eta(0)$ is the Navier-Stokes shear viscosity given by Eq. (4.10) and $\eta_{B}=\frac{1}{2} n \chi_{e}$ is the low density Boltzmann viscosity in the current units. The viscometric functions are

$$
\begin{gathered}
\Psi_{1}(a)=-n\left[1+\frac{8 \pi}{15} n^{*} \chi_{e}+\frac{16 \pi^{2}}{225}\left(n^{*} \chi_{e}\right)^{2}\right]+O\left(a^{2}\right) \\
\Psi_{2}(a)=-n \frac{64 \pi}{525}\left(n^{*} \chi_{e}\right)^{2}\left(3+\frac{8 \pi}{5} n^{*} \chi_{e}\right)+O\left(a^{2}\right)
\end{gathered}
$$

Finally, the shear dilatancy is

$$
\gamma(a)=n \frac{32 \pi}{225}\left(n^{*} \chi_{e}\right)^{2}\left(1+\frac{16 \pi}{15} n^{*} \chi_{e}\right)+O\left(a^{2}\right)
$$

The density dependence of these nonlinear transport coefficients is a nontrivial prediction of the kinetic model. Until recently, only the low density limit of these coefficients was known from kinetic theory. However, they now have been calculated from the RET at finite densities using Grad's moment method approximation, showing good agreement with results from computer simulation [7]. Comparison of the above expressions with those from the RET shows good agreement. In both cases, the kinetic contribution $\Psi_{1}^{k}(0)$ and $\gamma(0) /\left(n^{*} \chi_{e}\right)^{2}$ are linear functions of $n^{*} \chi_{e}$, the kinetic contribution $\Psi_{2}^{k}(0)$ is a quadratic function of $n^{*} \chi_{e}, \Psi_{2}(0)$ is a cubic function of $n^{*} \chi_{e}$, and $\Psi_{1}(0) \propto\left[\Psi_{1}^{k}(0)\right]^{2}$. To perform a more quantitative comparison, consider the extreme density of $n^{*} \simeq 0.95$, at which the hard-sphere system undergoes a fluid-solid transition [24]. According to the CarnahanStarling equation of state [19], this corresponds to $n^{*} \chi_{e}$ $\simeq 5$.6. The relative deviations of the model predictions for $\Psi_{1}(0), \Psi_{2}(0)$, and $\gamma(0)$ are found to be $1.2 \%, 13 \%$, and $2.1 \%$, respectively. Evidently, the kinetic model captures the density dependence of the RET very well for these leading order nonlinear transport coefficients.

The shear viscosity has been calculated to one order higher in the shear rate in Eq. (5.21) than the viscometric functions and it appears there have been no corresponding calculations from the RET as yet. At low and intermediate densities, the coefficient of $a^{2}$ in Eq. (5.21) is negative. This represents shear thinning, i.e., the shear viscosity decreases as the shear rate increases. Nevertheless, the term proportional to $\left(n^{*} \chi_{e}\right)^{4}$ has a sign opposite to that of the remaining terms. Consequently, there is a qualitative change at higher densities from shear thinning to shear thickening. This change takes place at $n^{*} \chi_{e} \simeq 3.06$, which corresponds to $n^{*} \simeq 0.79$. 


\section{B. Solution for large shear rates}

To study the case of asymptotically large values of the shear rate, Eq. (C11) can be rewritten as

$$
\begin{aligned}
\mathrm{P}^{c}= & \frac{m}{2} \sigma^{3} \chi_{e} \int d \hat{\boldsymbol{\sigma}} \hat{\boldsymbol{\sigma}} \hat{\boldsymbol{\sigma}} \int d \mathbf{V}_{1} \int d \mathbf{V}_{2} \Theta(\hat{\boldsymbol{\sigma}} \cdot(\mathbf{g}-\mathbf{a} \cdot \boldsymbol{\sigma})) \\
& \times[\hat{\boldsymbol{\sigma}} \cdot(\mathbf{g}-\mathrm{a} \cdot \boldsymbol{\sigma})]^{2} f\left(\mathbf{V}_{1}\right) f\left(\mathbf{V}_{2}\right),
\end{aligned}
$$

where the change of variables $\mathbf{V}_{1} \rightarrow \mathbf{V}_{1}-\mathbf{a} \cdot \boldsymbol{\sigma}$ has been made. In the limit $a \rightarrow \infty$ it is possible to neglect $\mathbf{g}$ relative to a. $\boldsymbol{\sigma}$, since the effective values of the relative velocity are not much larger than the thermal velocity. Consequently,

$$
\mathrm{P}^{c} \approx \frac{m}{2} \sigma^{3} \chi_{e} n^{2} a^{2} \sigma^{2} \int d \hat{\boldsymbol{\sigma}} \hat{\boldsymbol{\sigma}} \hat{\boldsymbol{\sigma}} \Theta\left(-\sigma_{x} \sigma_{y}\right) \sigma_{x}^{2} \sigma_{y}^{2},
$$

where use has been made of Eq. (2.3). More explicitly

$$
\begin{gathered}
P_{x x}^{c} \approx P_{y y}^{c} \approx 3 P_{z z}^{c} \approx n \frac{128 \pi^{2}}{875}\left(n^{*} \chi_{e}\right)^{3} a^{2}, \\
P_{x y}^{c} \approx-n \frac{1024 \pi}{2625}\left(n^{*} \chi_{e}\right)^{3} a^{2} .
\end{gathered}
$$

Also, the nonvanishing elements of $\mathrm{A}(a)$ and $\alpha(a)$ are found from Eqs. (5.2), (5.3), and (5.9) to have the asymptotic forms

$$
\begin{gathered}
A_{x x}=A_{y y}=-\frac{1}{2} A_{z z} \approx \frac{2048 \pi}{23625}\left(n^{*} \chi_{e}\right)^{3} a^{3}, \\
A_{x y} \approx-\frac{256 \pi^{2}}{2625}\left(n^{*} \chi_{e}\right)^{3} a^{3}, \\
\alpha \approx \frac{2048 \pi}{7875}\left(n^{*} \chi_{e}\right)^{3} a^{3}+\frac{\pi}{8} a .
\end{gathered}
$$

These results specify all the parameters of the formal solution (5.7) so an explicit asymptotic form of the distribution function is obtained.

It is shown in Appendix $\mathrm{C}$ that the collisional transfer contributions dominate the kinetic parts of the pressure tensor at large shear rates, so that Eqs. (5.27) determine the asymptotic behavior of the shear viscosity, viscometric functions, and shear dilatancy

$$
\begin{gathered}
\eta(a) \rightarrow n \frac{1024 \pi}{2625}\left(n^{*} \chi_{e}\right)^{3} a, \\
\Psi_{1}(a) \rightarrow 0, \quad \Psi_{2}(a) \rightarrow-n \frac{256 \pi^{2}}{2625}\left(n^{*} \chi_{e}\right)^{3}, \\
\gamma(a) \rightarrow n \frac{128}{1125}\left(n^{*} \chi_{e}\right)^{3} .
\end{gathered}
$$

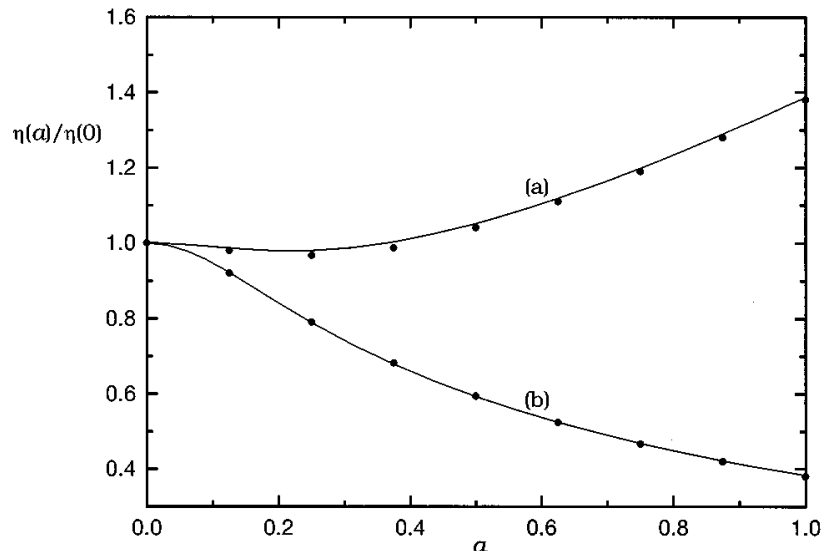

FIG. 1. Plot of (a) $\eta(a) / \eta(0)$ and (b) $\eta^{k}(a) / \eta^{k}(0)$ for $n^{*} \chi_{e}$ $=2$. Lines are from the kinetic model; symbols are from Monte Carlo simulations of the RET.

Equation (5.30) shows that shear thickening appears for any density at sufficiently large shear rates. The first viscometric function vanishes while $\Psi_{2}(a)$ and $\gamma(a)$ are qualitatively similar to their behavior at small shear rates.

\section{Comparison with Monte Carlo simulation}

As discussed in the Introduction, recent methods have been developed by two of us for Monte Carlo simulation of the solution to the RET kinetic equation [8]. These methods have been implemented for uniform shear flow to calculate the distribution function and rheological properties for comparison with the results from the kinetic model. The Monte Carlo method employed in this paper takes the uniformity condition $f(\mathbf{r}, \mathbf{v}, t)=f(\mathbf{V})$ for granted [25], so that the possible instability of the USF is not addressed. The results presented in this section demonstrate the utility of both the Monte Carlo and kinetic model for describing states far from equilibrium at dense fluid conditions accessible previously only via molecular dynamics simulation. The full solution to the kinetic model for arbitrary shear rates requires substitution of the formal solution (5.7) into Eq. (5.10) for $P_{x y}^{c}(a)$. While the velocity integrals are explicit and Gaussian, the remaining integrals pose a multidimension task that is difficult to implement in the self-consistent calculation of $P_{x y}^{c}(a)$ and $\alpha(a)$. Instead, we obtain a reasonable estimate for $P_{x y}^{c}(a)$ by using a first Sonine approximation for $f$ in Eq. (5.10),

$$
f \rightarrow f \curlywedge[1+\mathrm{C}: \mathrm{D}(V)]
$$

The coefficient $\mathrm{C}$ can be identified by requiring that the moments of this trial function with respect to $V_{i} V_{j}$ should be the same as those for the exact distribution,

$$
\mathrm{C}=\left(2 n^{-1} \mathrm{P}^{k}-1\right)
$$

With this approximation, the evaluation of $\mathrm{P}^{c}$ is similar to that of the tensor $\mathrm{A}(a)$, 


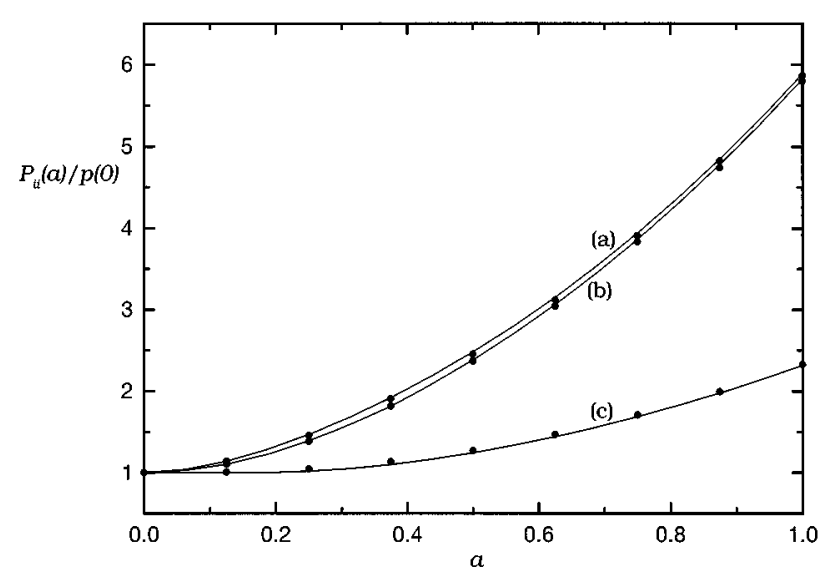

FIG. 2. Plot of (a) $P_{x x}(a) / p(0)$, (b) $P_{y y}(a) / p(0)$, and (c) $P_{z z}(a) / p(0)$ for $n^{*} \chi_{e}=2$. Lines are from the kinetic model; symbols are from Monte Carlo simulations of the RET.

$$
\begin{aligned}
P_{i j}^{c}= & -\frac{n}{4} n^{*} \chi_{e} \int d \hat{\boldsymbol{\sigma}} \hat{\sigma}_{i} \hat{\sigma}_{j}\left\{\frac{2}{\pi^{1 / 2}} \bar{a} \hat{\sigma}_{x} \hat{\sigma}_{y} e^{-\bar{a}^{2} \hat{\sigma}_{x}^{2} \hat{\sigma}_{y}^{2}}\right. \\
& -\left(1+2 \bar{a}^{2} \hat{\sigma}_{x}^{2} \hat{\sigma}_{y}^{2}\right) \operatorname{erfc}\left(\bar{a} \hat{\sigma}_{x} \hat{\sigma}_{y}\right) \\
& -\left(\hat{\boldsymbol{\sigma}} \hat{\boldsymbol{\sigma}}-\frac{1}{3} 1\right): \mathrm{C} \operatorname{erfc}\left(\bar{a} \hat{\sigma}_{x} \hat{\sigma}_{y}\right) \\
& \left.-\frac{1}{8}\left[\left(\hat{\boldsymbol{\sigma}} \hat{\boldsymbol{\sigma}}-\frac{1}{3} 1\right): \mathrm{C}\right]^{2} \frac{2}{\pi^{1 / 2}} \bar{a} \hat{\sigma}_{x} \hat{\sigma}_{y} e^{-\bar{a}^{2}} \hat{\sigma}_{x}^{2} \hat{\sigma}_{y}^{2}\right\} .
\end{aligned}
$$

The self-consistent determination of $P_{x y}^{c}(a)$ for a given density and $a$ is now a straightforward numerical iteration problem. As a check of this practical approach it is found that the small shear rate behavior is quite good, with exact results through second order in the shear rate. The super-Burnett coefficient $P_{x y}^{c(3)}$ is found to have the same qualitative density dependence and still shows shear thickening above a critical density, now given by $n^{*} \simeq 0.78$ instead of the more accurate value from Eq. (5.21) of $n^{*} \simeq 0.79$. The large shear rate be-

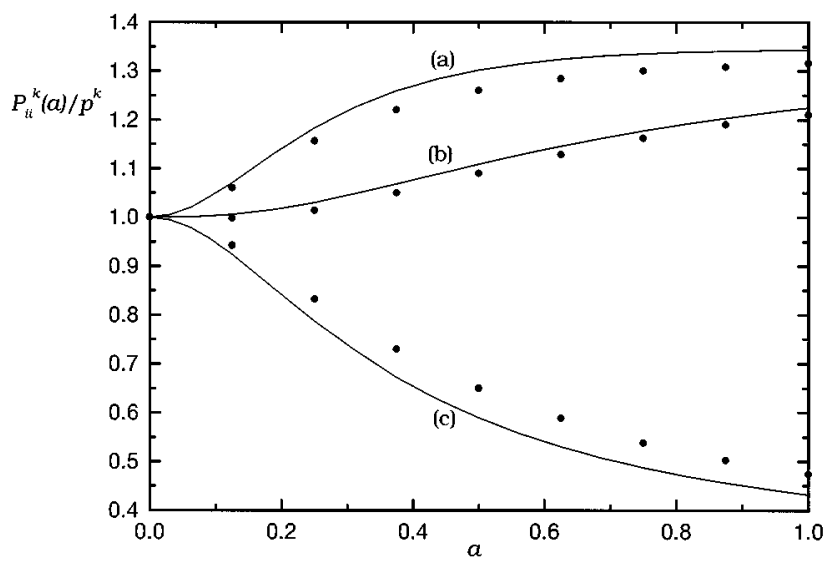

FIG. 3. Plot of (a) $P_{x x}^{k}(a) / p^{k}$, (b) $P_{y y}^{k}(a) / p^{k}$, and (c) $P_{z z}^{k}(a) / p^{k}$ for $n^{*} \chi_{e}=2$. Lines are from the kinetic model; symbols are from Monte Carlo simulations of the RET.

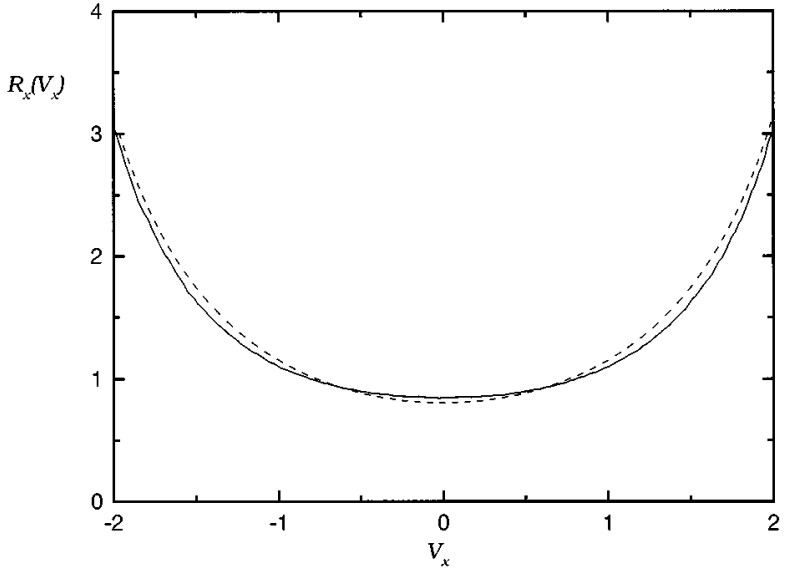

FIG. 4. Distribution function $R_{x}\left(V_{x}\right)$ for $n^{*} \chi_{e}=0.2$ and $a=1$. The dashed line is from the kinetic model; the solid line is from Monte Carlo simulations of the RET.

havior of $P_{x y}^{c}(a)$ depends only on the normalization of $f$ and therefore is the same as described above.

With $P_{x y}^{c}(a)$ and $\alpha(a)$ determined self-consistently in this way, the parameters of the formal distribution function (5.7) are known and any property of interest can be calculated from it, for arbitrary density and shear rate. Figure 1 shows a comparison of $\eta(a) / \eta(0)$ as a function of the shear rate for the high density of $n^{*} \chi_{e}=2$. Also shown is the corresponding kinetic part. The good agreement indicates that both the kinetic and collisional transfer contributions are given accurately by the model. The chosen density is such that the shear thickening effect due to the collisional transfer parts dominates for shear rates above about 0.3. Figures 2 and 3 show the normal stresses $P_{x x}(a) / p(0)$, etc., as a function of the shear rate at the same density. While there are noticeable differences for the kinetic parts for the $x x$ and $z z$ components, the total normal stress is given quite accurately in all cases. A similar accuracy holds at low density so we conclude that the kinetic model gives a good description of rheological transport throughout the density-shear rate plane.

To test the distribution function itself, we consider the reduced distributions for a single component of the velocity

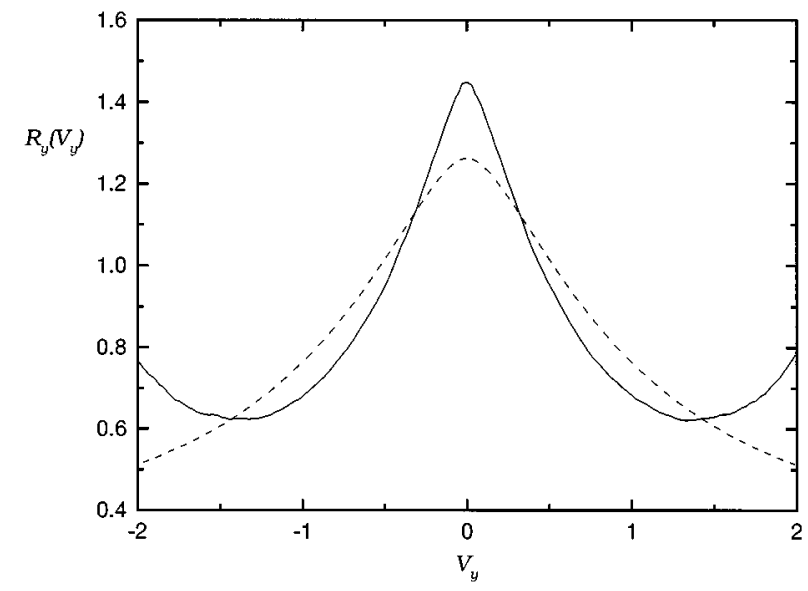

FIG. 5. The same as in Fig. 4 but for the distribution function $R_{y}\left(V_{y}\right)$. 


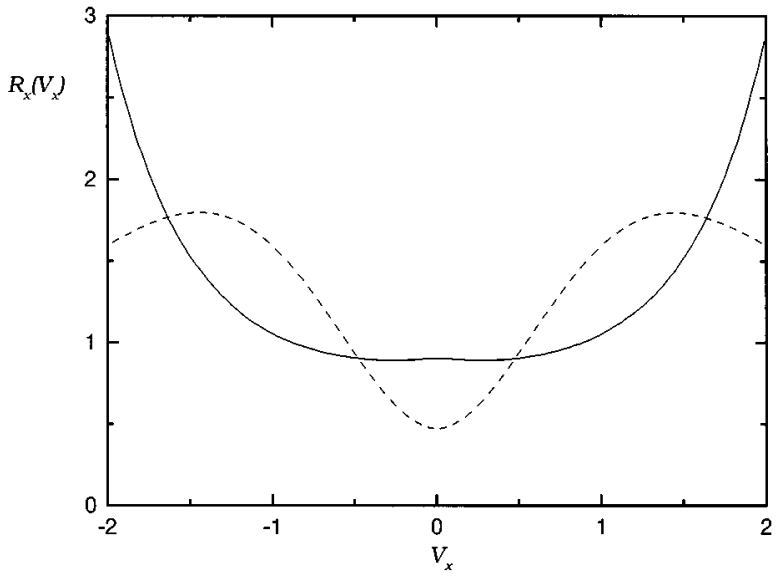

FIG. 6. The same as in Fig. 4 but for $n^{*} \chi_{e}=0.5$.

relative to the corresponding local equilibrium distribution, e.g.,

$$
R_{x}\left(V_{x}\right)=\frac{\int d V_{y} d V_{z} f(\mathbf{V})}{\int d V_{y} d V_{z} f(\mathbf{V})},
$$

with similar definitions for the $y$ and $z$ component distributions. Since they are defined relative to the local equilibrium distribution, they represent distortions that occur only far from equilibrium. As an extreme case we consider $a=1$. In the chosen units this is a shear rate equal to the collision frequency, which is very large, particularly at high density. Figure 4 shows $R_{x}$ for the moderate density of $n^{*} \chi_{e}=0.2$.

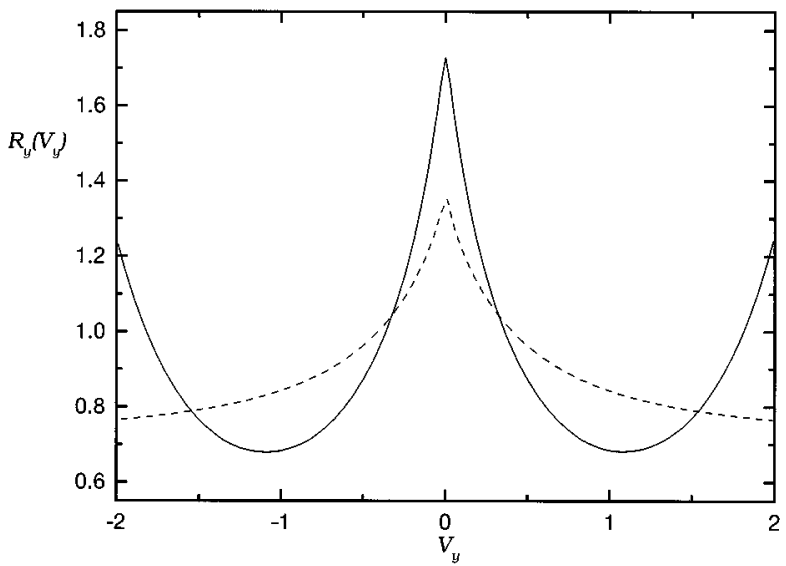

FIG. 7. The same as in Fig. 5 but for $n^{*} \chi_{e}=0.5$.

The agreement is exceptionally good for this highly nonequilibrium state. Figure 5 shows the same comparison for $R_{y}$. The agreement is less striking but the qualitative features are preserved by the kinetic model, except for velocities exceeding the thermal velocity. Figures 6 and 7 show the corresponding comparisons at the higher density of $n^{*} \chi_{e}=0.5$, still for $a=1$. This corresponds to a shear rate for which the collisional part of the shear viscosity is about $50 \%$ larger than its kinetic part. The predictions of the model are now less reliable, with even the qualitative features of $R_{x}$ given incorrectly, although the model correctly predicts a cusp of $R_{y}$ at $V_{y}=0$. At sufficiently large density and/or shear rate, the distribution function is no longer positive. To understand this failure, consider the behavior of $R_{y}$ for small $\left|V_{y}\right|$ :

$$
R_{y}\left(V_{y}\right) \approx\left\{\begin{array}{l}
\frac{1-A_{x x}-\frac{2}{3} n^{-1} a^{2} \eta^{c}(a)}{1-\frac{2}{3} n^{-1} a^{2} \eta(a)} \quad(\alpha<1), \\
\frac{\Gamma\left(\frac{1}{2}-\frac{1}{2 \alpha}\right)}{2 \alpha}\left(1-\frac{A_{x x}+\frac{2}{3} n^{-1} a^{2} \eta^{c}(a)}{\frac{2}{3} n^{-1} a^{2} \eta(a)}\right)\left|V_{y}\right|^{-\left(1-\alpha^{-1}\right)} \quad(\alpha>1) .
\end{array}\right.
$$

Thus $R_{y}\left(V_{y}\right)<0$ for small $\left|V_{y}\right|$ whenever $A_{x x}$ $>\frac{2}{3} n^{-1} a^{2} \eta^{k}(a)$. This failure of the model originates from the approximation to $(1-\mathcal{P}) \delta \nu f=(1-\mathcal{P}) J_{E}\left[f_{\ell}\right][$ see above Eq. (3.11)], which leads to an anomalous velocity dependence under these extreme conditions. However, this approximation does not affect the moment equation (5.8), so the transport properties are still given accurately. If a better velocity dependence is required, direct calculation of (1 $-\mathcal{P}) J_{E}\left[f_{\ell}\right]$ is possible for this case of uniform shear flow.

\section{DISCUSSION}

The revised Enskog kinetic theory for hard spheres provides a firm theoretical basis to address a wide range of nonequilibrium phenomena for both fluids and solids, near and far from equilibrium. Recently developed Monte Carlo methods provide the first general means for practical solution to the RET in this broad context. In the present work we have presented a complementary analytical method, using a model kinetic equation closely related to the RET. These two methods have great potential for a new understanding of nonequilibrium systems under conditions accessible previously only by molecular dynamics simulation. Although the RET is limited to hard spheres, many of the physical mechanisms active in more realistic systems occur for hard spheres as well.

The Chapman-Enskog expansion of Sec. IV shows that the kinetic model captures the correct density dependence of 
the RET for transport near equilibrium. This prediction of density dependence is further reinforced by the exact agreement for the nonlinear transport coefficients considered in Sec. V. The most severe test of the model here has been its prediction of the shear rate dependence of the pressure tensor at high densities, far from equilibrium. Figures 1-3 illustrate the impressive predictive quality under such conditions for both the Monte Carlo simulation method and the kinetic model. This preliminary study of shear flow already has given new insight into the competition between kinetic and collisional transfer mechanisms. For example, it has been noted that the collisional transfer leads to a crossover from shear thinning to shear thickening at sufficiently large shear rates. In this study we have not addressed the stability of uniform shear flow and it is possible that the large shear rate domain considered represents metastable states. It is well established that there is a transition at large shear rates and high densities to an ordered phase which may occur before shear thickening effects dominate. Although it has been suggested that this transition is due to a hydrodynamic instability, and therefore should be predicted by the RET, it has not been seen from the Monte Carlo simulations yet. If some other mechanism is responsible for the transition it is possible that this is not contained in the RET. Further Monte Carlo studies are required to resolve this question.

There is a class of exact solutions to the BGK kinetic model at low density for various types of heat and momentum transport. It is expected that the kinetic model proposed here for the RET can be solved for this class at the same level as described here for uniform shear flow. More interesting perhaps are the new problems relevant only at higher densities. These include flow of two immiscible fluids near a solid boundary where the usual boundary conditions fail at the contact line [26]. This and related boundary layer problems have been studied recently by molecular dynamics simulation but without other theoretical analysis. Other examples of interest occur in the crystal phase, for which there have been few studies to date. Extensions of hydrodynamic descriptions to short wavelengths have been very successful in the fluid phase, and it is expected a similar description based on local elastic constants could be developed for the crystal phase as well using the simplifications afforded by the kinetic model. Finally, there is a new possibility to study the dynamics of metastable fluid and crystal states from a fundamental kinetic theory point of view. Since the theory contains the structural features of metastable states in $\chi[n]$ (the free energy topology), such effects as structural arrest for selected initial conditions (e.g., the wrong crystal structure, quenched fluid) can be considered. The latter are relevant for understanding a possible hard-sphere glass transition and the kinetics of a disordered crystal (vacancy diffusion). We plan to address some of these topics in the near future.

Finally, we note that both the RET and the kinetic model presented here have been extended to the case of inelastic hard-sphere collisions $[10,11]$. The corresponding ChapmanEnskog solution for the kinetic model has been used to derive hydrodynamic equations and transport coefficients for rapid granular flow. Such calculations are prohibitively difficult from the RET, without further uncontrolled approximations, but are straightforward from the kinetic model. Appli- cation to uniform shear flow at low density also has shown good agreement with direct numerical simulation [27], so it is expected that its extension to the dense fluid case considered here also yield the same level of accuracy.

\section{ACKNOWLEDGMENTS}

The research of J.W.D. was supported by NSF Grant No. PHY 9722133. Partial support by the Dirección General de Investigación Científica y Técnica (Spain) through Grant Nos. PB96-0534 (J.J.B.) and PB94-1021 (J.M.M. and A.S.) is gratefully acknowledged.

\section{APPENDIX A: KINETIC MODELING AND MOMENT METHODS}

In this appendix a brief overview of kinetic modeling and moment methods is provided to put in context and to further motivate the specific choice made here. The general kinetic equation is assumed to have the form

$$
\left(\partial_{t}+\mathbf{v} \cdot \nabla\right) f(\mathbf{r}, \mathbf{v} ; t)=J[\mathbf{r}, \mathbf{v} \mid f(t)],
$$

where $J[\mathbf{r}, \mathbf{v} \mid f(t)]$ denotes the collision operator, specified as a given functional of $f$ that is local in $\mathbf{r}$ and $t$. The discussion here can be generalized to include an external force and to collision operators that are nonlocal functionals.

\section{Moment methods}

The simplest class of approximate solutions are those obtained by a representation of $f$ as a vector in a Hilbert space spanned by a complete set of functions $\left\{\zeta_{\alpha}(\mathbf{v})\right\}$,

$$
\begin{gathered}
f(\mathbf{r}, \mathbf{v} ; t)=\sum_{\alpha} c_{\alpha}(\mathbf{r}, t) \zeta_{\alpha}(\mathbf{v}), \\
c_{\alpha}(\mathbf{r}, t)=\int d \mathbf{v} \psi_{\alpha}^{*}(\mathbf{v}) f(\mathbf{r}, \mathbf{v} ; t),
\end{gathered}
$$

where $\left\{\psi_{\alpha}(\mathbf{v})\right\}$ is a corresponding biorthogonal set defined by $\int d \mathbf{v} \psi_{\alpha}^{*}(\mathbf{v}) \zeta_{\beta}(\mathbf{v})=\delta_{\alpha, \beta}$. Substitution of Eq. (A2) into Eq. (A1) gives an infinite set of coupled equations for the coefficients $c_{\alpha}(\mathbf{r}, t)$,

$$
\begin{gathered}
\partial_{t} c_{\alpha}(\mathbf{r}, t)+\mathbf{A}_{\alpha \beta} \cdot \nabla c_{\beta}(\mathbf{r}, t)=J_{\alpha}[\mathbf{r}, t \mid f], \\
\mathbf{A}_{\alpha \beta}=\int d \mathbf{v} \psi_{\alpha}^{*}(\mathbf{v}) \mathbf{v} \zeta_{\beta}(\mathbf{v}), \\
J_{\alpha}[\mathbf{r}, t \mid f]=\int d \mathbf{v} \psi_{\alpha}^{*}(\mathbf{v}) J[\mathbf{r}, \mathbf{v} \mid f(t)] .
\end{gathered}
$$

There is little advantage to this representation unless the matrix $\mathbf{A}_{\alpha \beta}$ is sparse, i.e., couples only few pairs of functions, and if $J_{\alpha}$ depends only on relatively few coefficients $c_{\gamma}$. Even in this case approximations are required to close the infinite set of coupled equations. These approximations entail a restriction of the sum in Eq. (A2) to some finite set $\left\{\zeta_{\alpha} ; \alpha \in \mathcal{S}\right\}$ and implies that the solution is being approximated in a corresponding finite dimensional subspace of the Hilbert space. The choice of this set is suggested by the average properties of interest, to ensure that their prediction 
is included in the approximation considered. Otherwise, the selection of functions $\left\{\zeta_{\alpha}(\mathbf{v})\right\}$ and subset $\alpha \in \mathcal{S}$ requires further analysis of the convergence properties or successive approximations. The best studied example is that due to Grad who chose Hermite polynomials for $\left\{\psi_{\alpha}(\mathbf{v})\right\}$ and chose as the approximate finite set those polynomials in the velocity for which the corresponding $c_{\alpha}(\mathbf{r}, t)$ are the average mass density, energy density, momentum density, pressure tensor, and heat flux. This 13 moment approximation is reasonable since his interests were in the derivation of hydrodynamics from the conservation laws which relate just these fields.

A variant of this method is to approximate only the collision operator by an expansion in a finite subset of the $\left\{\zeta_{\alpha}(\mathbf{v})\right\}$ :

$$
\left(\partial_{t}+\mathbf{v} \cdot \nabla\right) f(\mathbf{r}, \mathbf{v} ; t)=\sum_{\alpha \in \mathcal{S}} \zeta_{\alpha}(\mathbf{v}) J_{\alpha}[\mathbf{r}, t \mid f] .
$$

In this case, the distribution function is not restricted to a finite dimensional subspace, but rather is determined by the solution to Eq. (A5). The solution is obtained in two steps. First $f(\mathbf{r}, \mathbf{v} ; t)$ is determined (trivially) as a linear functional of $J_{\alpha}[\mathbf{r}, t \mid f]$. Next, a nonlinear integral equation for $J_{\alpha}[\mathbf{r}, t \mid f]$ is obtained from this solution using the definition of $J_{\alpha}$. These two sets of equations must be solved selfconsistently. The advantage is that the nonlinear integral equation no longer depends on the velocity. This choice of approximating the collision operator rather than the solution to the kinetic equation is the basic difference between the moment methods and kinetic models. Equation (A5) is one example of a kinetic model.

\section{Linear kinetic models}

The construction of kinetic models is most straightforward for linear kinetic theories. Consider some known reference state $f_{0}$ and consider small perturbations from this state $f=f_{0}+\delta f$. Then, retaining terms in Eq. (A1) only to linear order in the small perturbation gives

$$
\left(\partial_{t}+\mathbf{v} \cdot \nabla\right) \delta f(\mathbf{r}, \mathbf{v} ; t)=I(\mathbf{r}, \mathbf{v} ; t)+L[\mathbf{r}, \mathbf{v} \mid \delta f(t)],
$$

where $I=J\left[f_{0}\right]-\left(\partial_{t}+\mathbf{v} \cdot \nabla\right) f_{0}$ and $L$ is the linear functional of $\delta f$ obtained from $J\left[f_{0}+\delta f\right]$. To construct a kinetic model the linear functional is first expanded in the complete set of functions

$$
\begin{gathered}
L[\mathbf{r}, \mathbf{v} \mid \delta f(t)]=\sum_{\alpha, \beta} \zeta_{\alpha}(\mathbf{v}) L_{\alpha \beta} \delta c_{\beta}(\mathbf{r}, t), \\
L_{\alpha \beta}=\int d \mathbf{v} \psi_{\alpha}^{*}(\mathbf{v}) L\left[\mathbf{r}, \mathbf{v} \mid \zeta_{\beta}\right] .
\end{gathered}
$$

This is still an exact representation of $L$. Now separate off the contribution to the double sum from $\alpha, \beta \in \mathcal{S}$ and make the approximation $L_{\alpha \beta} \rightarrow-\lambda \delta_{\alpha, \beta}$ for the $\alpha, \beta \notin \mathcal{S}$,

$$
\begin{aligned}
L[\mathbf{r}, \mathbf{v} \mid \delta f(t)] \rightarrow & \sum_{\alpha, \beta \in \mathcal{S}} \zeta_{\alpha}(\mathbf{v}) L_{\alpha \beta} \delta c_{\beta}(\mathbf{r}, t) \\
& -\lambda \sum_{\alpha \notin \mathcal{S}} \zeta_{\alpha}(\mathbf{v}) \delta c_{\alpha}(\mathbf{r}, t) \\
= & \sum_{\alpha, \beta \in \mathcal{S}} \zeta_{\alpha}(\mathbf{v})\left(L_{\alpha \beta}+\lambda \delta_{\alpha, \beta}\right) \delta c_{\beta}(\mathbf{r}, t) \\
& -\lambda \sum_{\alpha} \zeta_{\alpha}(\mathbf{v}) \delta c_{\alpha}(\mathbf{r}, t) .
\end{aligned}
$$

The summation in the last term of Eq. (A8) extends over the complete set and is therefore simply $\delta f$. The linear kinetic equation with this approximation becomes

$$
\begin{aligned}
\left(\partial_{t}\right. & +\mathbf{v} \cdot \nabla+\lambda) \delta f(\mathbf{r}, \mathbf{v} ; t) \\
& =I(\mathbf{r}, \mathbf{v} ; t)+\sum_{\alpha, \beta \in \mathcal{S}} \zeta_{\alpha}(\mathbf{v})\left(L_{\alpha \beta}+\lambda \delta_{\alpha, \beta}\right) \delta c_{\beta}(\mathbf{r}, t) .
\end{aligned}
$$

This equation can be solved in the same self-consistent way as Eq. (A5). The proportionality constant $\lambda$ is clearly independent of velocity from this analysis, but can be chosen as one of the matrix elements in the complementary subspace, chosen to fit some property (e.g., a transport coefficient), or to otherwise optimize the approximation. In other contexts (percolation theory, lattice gases) closely related approximations are called effective medium theories.

The linear operator is approximated such that it is exact in the subspace defined by $\mathcal{S}$ and approximated elsewhere simply as proportional to the identity operator. This can be summarized in terms of the projection operator $\mathcal{P}_{0}$ onto this subspace,

$$
\begin{gathered}
\mathcal{L}=\mathcal{P}_{0} \mathcal{L} \mathcal{P}_{0}-\lambda\left(1-\mathcal{P}_{0}\right), \\
\mathcal{P}_{0} X \equiv \sum_{\alpha \in \mathcal{S}} \zeta_{\alpha}(\mathbf{v}) \int d \mathbf{v} \psi_{\alpha}^{*}(\mathbf{v}) X(\mathbf{v}),
\end{gathered}
$$

where $\mathcal{L}$ denotes the linear operator associated with $L$.

\section{Nonlinear kinetic models}

The linear kinetic models are based on a matrix representation of the linear collision operator that is preserved exactly in some subspace and approximated in its complement. Clearly, it can be improved systematically by expanding the dimension of the chosen subspace. For nonlinear collision operators this decomposition into its contributions in two complementary subspaces also is possible,

$$
\left(\partial_{t}+\mathbf{v} \cdot \nabla\right) f=\mathcal{P} J+(1-\mathcal{P}) J,
$$

where $\mathcal{P}$ is the projection operator onto the subspace for $\alpha$ $\in \mathcal{S}$ defined in the same way as $\mathcal{P}_{0}$ [the notation is changed to distinguish the choices of $\left\{\zeta_{\alpha}(\mathbf{v})\right\}$ for linear and nonlinear models, as indicated below]. The approximation (A5) corresponds to neglecting the last term on the right side of Eq. (A11). Instead, it is better to retain some approximation to this contribution. The choice analogous to that of Eq. (A10) 
for linear operators is to approximate the collision operator in this subspace as proportional to the identity operator, (1 $-\mathcal{P}) J \rightarrow-(1-\mathcal{P}) \lambda f$. In contrast to the linear case, we now allow $\left\{\zeta_{\alpha}(\mathbf{v})\right\}, \mathcal{P}$, and $\lambda$ to be functionals of $f$. This gives a great deal more flexibility in the construction of kinetic models, at the price of being less systematic. Let $f_{0}$ denote a distribution function that is a functional of $f$ through the constraints that they have the same average values of $\psi_{\alpha}^{*}(\mathbf{v})$ for $\alpha \in \mathcal{S}$,

$$
\int d \mathbf{v} \psi_{\alpha}^{*}(\mathbf{v})\left(f(\mathbf{v})-f_{0}[\mathbf{v} \mid f]\right)=0
$$

and choose the basis set to be $\left\{\zeta_{\alpha}(\mathbf{v})\right\}=\left\{f_{0} \psi_{\alpha}(\mathbf{v})\right\}$. Also, let $\lambda[\mathbf{v} \mid f]=\nu[f]+\delta \nu[\mathbf{v} \mid f]$, where $\nu[f]$ is independent of the velocity. The kinetic model (A11) becomes

$$
\left(\partial_{t}+\mathbf{v} \cdot \nabla\right) f=-\nu\left(f-f_{0}\right)+\mathcal{P} J-(1-\mathcal{P}) \delta \nu f,
$$

where we have assumed that $\psi_{\alpha}=1$ belongs to the set $\alpha$ $\in \mathcal{S}$, which implies $\mathcal{P} f=f_{0}$. This kinetic model has two velocity dependent functionals, $f_{0}$ and $\delta \nu$, and one velocity independent functional, $\nu$, as free parameters to optimize simplicity and accuracy. Clearly, a wide range of constraints can be accommodated in such a structure. The emphasis on hydrodynamics and transport in the text suggests the choice of subspace to be that spanned by polynomials in the velocity corresponding to the conserved densities. Then Eq. (A13) necessarily yields the exact conservation laws. The use of $f_{0} \rightarrow f_{\ell}$ is a convenient way to incorporate the condition that $f_{0}\left[\mathbf{v} \mid f_{e}\right]=f_{e}$ is the stationary equilibrium distribution, but other choices are possible as well. Finally, the choices for $\nu$ and $\delta \nu$ have been selected for accuracy of transport coefficients. As seen in Sec. V, better choices may be necessary for the distribution of velocities under extreme conditions.

Finally, the connection of this work with that of Ref. [7] is considered. There are two components to that work, first a moment method approximation and then a kinetic model is proposed to improve that approximation. The moment method approximation is given by a straightforward application of Grad's method: $f \rightarrow \mathcal{P} f, J[f] \rightarrow \mathcal{P} J[\mathcal{P} f]$, resulting in a closed set of equations obtained from Eq. (A3) in the subspace of 13 velocity moments. To improve this approximation a kinetic model is proposed, not for $J[f]$ but for $J[f]$ $-J\left[f_{\ell}\right]$ :

$$
\begin{aligned}
J[f] & =J\left[f_{\ell}\right]+\mathcal{P}\left(J[f]-J\left[f_{\ell}\right]\right)+(1-\mathcal{P})\left(J[f]-J\left[f_{\ell}\right]\right) \\
& \rightarrow J\left[f_{\ell}\right]+\mathcal{P}\left(J[f]-J\left[f_{\ell}\right]\right)-(1-\mathcal{P}) \nu f \\
& =-\nu\left(f-f_{\ell}\right)+\mathcal{P} J[f]+(1-\mathcal{P}) J\left[f_{\ell}\right] \\
& \rightarrow-\nu\left(f-f_{\ell}\right)+\mathcal{P} J[\mathcal{P} f]+(1-\mathcal{P}) J\left[f_{\ell}\right] .
\end{aligned}
$$

The first approximation on the second line of Eq. (A14) corresponds to the choice $\delta \nu f=-J\left[f_{\ell}\right]$, which is the same as Eq. (3.9). Hence, this model is more general than that considered here which retains only certain projections of this result for greater simplicity. The approximation in the fourth line of Eq. (A14) retains only those restricted contributions in the subspace $\mathcal{P}$ that are retained in the moment approximation. In this respect it is more limited than the approximation considered here. Thus two different approximations are made, the first leading to more accurate but more complex structure while the second leads to greater simplicity but less accuracy. These differences illustrate the flexibility of kinetic modeling to adapt to both mathematical and subjective constraints. In the present case, both models, that given here and in [7] appear to give surprisingly good representations of the RET.

\section{APPENDIX B: COLLISIONAL TRANSFER CONTRIBUTIONS}

In this appendix some details of the derivation of Eqs. (2.15) and (2.16) are given. Let $\xi(\mathbf{v})$ be an arbitrary function of $\mathbf{v}$ and consider the integral

$$
\begin{aligned}
I_{\xi} \equiv & \int d \mathbf{v}_{1} \xi\left(\mathbf{v}_{1}\right) J_{E}\left[\mathbf{r}_{1}, \mathbf{v}_{1} \mid f(t)\right] \\
= & \sigma^{2} \int d \mathbf{v}_{1} \int d \mathbf{v}_{2} \int d \hat{\boldsymbol{\sigma}} \Theta(\hat{\boldsymbol{\sigma}} \cdot \mathbf{g})(\hat{\boldsymbol{\sigma}} \cdot \mathbf{g}) \xi\left(\mathbf{v}_{1}\right) \\
& \times\left\{\chi\left[\mathbf{r}_{1}, \mathbf{r}_{1}-\boldsymbol{\sigma} \mid n(t)\right] f\left(\mathbf{r}_{1}, \mathbf{v}_{1}^{\prime}, t\right) f\left(\mathbf{r}_{1}-\boldsymbol{\sigma}, \mathbf{v}_{2}^{\prime}, t\right)\right. \\
& \left.-\chi\left[\mathbf{r}_{1}, \mathbf{r}_{1}+\boldsymbol{\sigma} \mid n(t)\right] f\left(\mathbf{r}_{1}, \mathbf{v}_{1}, t\right) f\left(\mathbf{r}_{1}+\boldsymbol{\sigma}, \mathbf{v}_{2}, t\right)\right\}
\end{aligned}
$$

By making the changes $\left(\mathbf{v}_{1}, \mathbf{v}_{2}\right) \rightarrow\left(\mathbf{v}_{1}^{\prime}, \mathbf{v}_{2}^{\prime}\right)$ and $\hat{\boldsymbol{\sigma}} \rightarrow-\hat{\boldsymbol{\sigma}}$ in the first term of the right hand side, one gets

$$
\begin{aligned}
I_{\xi}= & \sigma^{2} \int d \mathbf{v}_{1} \int d \mathbf{v}_{2} \int d \hat{\boldsymbol{\sigma}} \Theta(\hat{\boldsymbol{\sigma}} \cdot \mathbf{g})(\hat{\boldsymbol{\sigma}} \cdot \mathbf{g})\left[\xi\left(\mathbf{v}_{1}^{\prime}\right)\right. \\
& \left.-\xi\left(\mathbf{v}_{1}\right)\right] \chi\left[\mathbf{r}_{1}, \mathbf{r}_{1}+\boldsymbol{\sigma} \mid n(t)\right] f\left(\mathbf{r}_{1}, \mathbf{v}_{1}, t\right) f\left(\mathbf{r}_{1}+\boldsymbol{\sigma}, \mathbf{v}_{2}, t\right) .
\end{aligned}
$$

Now we take $\xi(\mathbf{v})$ to be one of the summational invariants, i.e., $\xi(\mathbf{v})=\left\{1, \mathbf{v}, v^{2}\right\}$. Then, it is $\xi\left(\mathbf{v}_{1}^{\prime}\right)-\xi\left(\mathbf{v}_{1}\right)=\xi\left(\mathbf{v}_{2}\right)$ $-\xi\left(\mathbf{v}_{2}^{\prime}\right)$, so that we can write

$$
\begin{aligned}
I_{\xi}= & \sigma^{2} \int d \mathbf{v}_{1} \int d \mathbf{v}_{2} \int d \hat{\boldsymbol{\sigma}} \Theta(\hat{\boldsymbol{\sigma}} \cdot \mathbf{g})(\hat{\boldsymbol{\sigma}} \cdot \mathbf{g})\left[\xi\left(\mathbf{v}_{2}\right)\right. \\
& \left.-\xi\left(\mathbf{v}_{2}^{\prime}\right)\right] \chi\left[\mathbf{r}_{1}, \mathbf{r}_{1}+\boldsymbol{\sigma} \mid n(t)\right] f\left(\mathbf{r}_{1}, \mathbf{v}_{1}, t\right) f\left(\mathbf{r}_{1}+\boldsymbol{\sigma}, \mathbf{v}_{2}, t\right) \\
= & \sigma^{2} \int d \mathbf{v}_{1} \int d \mathbf{v}_{2} \int d \hat{\boldsymbol{\sigma}} \Theta(\hat{\boldsymbol{\sigma}} \cdot \mathbf{g})(\hat{\boldsymbol{\sigma}} \cdot \mathbf{g})\left[\xi\left(\mathbf{v}_{1}\right)\right. \\
& \left.-\xi\left(\mathbf{v}_{1}^{\prime}\right)\right] \chi\left[\mathbf{r}_{1}, \mathbf{r}_{1}-\boldsymbol{\sigma} \mid n(t)\right] f\left(\mathbf{r}_{1}, \mathbf{v}_{2}, t\right) f\left(\mathbf{r}_{1}-\boldsymbol{\sigma}, \mathbf{v}_{1}, t\right),
\end{aligned}
$$

where in the last step we have performed the changes $\mathbf{v}_{1} \leftrightarrow \mathbf{v}_{2}$ and $\hat{\boldsymbol{\sigma}} \rightarrow-\hat{\boldsymbol{\sigma}}$. Combination of Eqs. (B2) and (B3) yields

$$
\begin{aligned}
I_{\xi}= & \frac{\sigma^{2}}{2} \int d \mathbf{v}_{1} \int d \mathbf{v}_{2} \int d \hat{\boldsymbol{\sigma}} \Theta(\hat{\boldsymbol{\sigma}} \cdot \mathbf{g})(\hat{\boldsymbol{\sigma}} \cdot \mathbf{g})\left[\xi\left(\mathbf{v}_{1}^{\prime}\right)-\xi\left(\mathbf{v}_{1}\right)\right] \\
& \times\left\{\chi\left[\mathbf{r}_{1}, \mathbf{r}_{1}+\boldsymbol{\sigma} \mid n(t)\right] f\left(\mathbf{r}_{1}, \mathbf{v}_{1}, t\right) f\left(\mathbf{r}_{1}+\boldsymbol{\sigma}, \mathbf{v}_{2}, t\right)-\chi\left[\mathbf{r}_{1}, \mathbf{r}_{1}\right.\right. \\
& \left.-\boldsymbol{\sigma} \mid n(t)] f\left(\mathbf{r}_{1}-\boldsymbol{\sigma}, \mathbf{v}_{1}, t\right) f\left(\mathbf{r}_{1}, \mathbf{v}_{2}, t\right)\right\} .
\end{aligned}
$$

Next, we note the identity 


$$
\begin{aligned}
& F(\mathbf{r}, \mathbf{r}+\boldsymbol{\sigma})-F(\mathbf{r}-\boldsymbol{\sigma}, \mathbf{r}) \\
& \quad=\int_{0}^{1} d \lambda \frac{\partial}{\partial \lambda} F(\mathbf{r}-(1-\lambda) \boldsymbol{\sigma}, \mathbf{r}+\lambda \boldsymbol{\sigma}) \\
& \quad=\boldsymbol{\sigma} \cdot \nabla \int_{0}^{1} d \lambda F(\mathbf{r}-(1-\lambda) \boldsymbol{\sigma}, \mathbf{r}+\lambda \boldsymbol{\sigma}) .
\end{aligned}
$$

By taking into account the property $\chi\left[\mathbf{r}_{1}, \mathbf{r}_{2} \mid n\right]=\chi\left[\mathbf{r}_{2}, \mathbf{r}_{1} \mid n\right]$, we can then rewrite Eq. (B4) as a divergence:

$$
\begin{aligned}
I_{\xi}= & -\nabla_{1} \cdot \frac{\sigma^{3}}{2} \int d \mathbf{v}_{1} \int d \mathbf{v}_{2} \int d \hat{\boldsymbol{\sigma}} \Theta(\hat{\boldsymbol{\sigma}} \cdot \mathbf{g})(\hat{\boldsymbol{\sigma}} \cdot \mathbf{g}) \hat{\boldsymbol{\sigma}}\left[\xi\left(\mathbf{v}_{1}\right)\right. \\
& \left.-\xi\left(\mathbf{v}_{1}^{\prime}\right)\right] \int_{0}^{1} d \lambda \chi\left[\mathbf{r}_{1}-(1-\lambda) \boldsymbol{\sigma}, \mathbf{r}_{1}+\lambda \boldsymbol{\sigma} \mid n(t)\right] \\
& \times f\left(\mathbf{r}_{1}-(1-\lambda) \boldsymbol{\sigma}, \mathbf{v}_{1}, t\right) f\left(\mathbf{r}_{1}+\lambda \boldsymbol{\sigma}, \mathbf{v}_{2}, t\right) .
\end{aligned}
$$

Particularizing this equation for $\xi(\mathbf{v})=m \mathbf{v}$, Eq. (2.15) follows directly. To get Eq. (2.16), choose $\xi(\mathbf{v})=(m / 2) v^{2}$ and use the relation

$$
\begin{aligned}
\frac{m}{2}\left(v_{1}^{2}-v_{1}^{\prime 2}\right) & =m(\hat{\boldsymbol{\sigma}} \cdot \mathbf{G})(\hat{\boldsymbol{\sigma}} \cdot \mathbf{g}) \\
& =m(\hat{\boldsymbol{\sigma}} \cdot \widetilde{\mathbf{G}})(\hat{\boldsymbol{\sigma}} \cdot \mathbf{g})+m(\hat{\boldsymbol{\sigma}} \cdot \mathbf{u})(\hat{\boldsymbol{\sigma}} \cdot \mathbf{g}),
\end{aligned}
$$

where $\mathbf{G}=\frac{1}{2}\left(\mathbf{v}_{1}+\mathbf{v}_{2}\right)$ and $\widetilde{\mathbf{G}}=\mathbf{G}-\mathbf{u}$.

\section{APPENDIX C: CALCULATION OF $\mathrm{P}^{k}(a)$ AND $\mathrm{P}^{c}(a)$}

In this appendix some further details for the calculation of the pressure tensor in Sec. V are given. Consider first the kinetic part $\mathrm{P}^{k}(a)$. Substitution of the formal solution (5.7) into Eq. (2.11) gives

$$
\begin{aligned}
\mathbf{P}^{k} & =\int d \mathbf{V} m \mathbf{V} \mathbf{V} \int_{0}^{\infty} d s e^{-(1-3 \alpha) s} \widetilde{f} /\left(e^{\alpha s}(1+s \mathbf{a}) \cdot \mathbf{V}\right) \\
& \left.=m \int_{0}^{\infty} d s e^{-(1+2 \alpha) s} \int d \mathbf{V} \widetilde{f} / \mathbf{V}\right)(1-s \mathbf{a}) \cdot \mathbf{V}(1-s \mathbf{a}) \cdot \mathbf{V}
\end{aligned}
$$

The integrals are easily performed to give the nonzero elements of the kinetic pressure tensor

$$
\begin{gathered}
P_{x x}^{k}=\frac{n}{2}(1+2 \alpha)^{-1}\left\{\left(1-\frac{4}{3} a n^{-1} P_{x y}^{c}+2 A_{x x}\right)\right. \\
\left.\quad \times\left[1+2(1+2 \alpha)^{-2} a^{2}\right]-4 a(1+2 \alpha)^{-1} A_{x y}\right\} \\
P_{y y}^{k}=\frac{n}{2}(1+2 \alpha)^{-1}\left(1-\frac{4}{3} a n^{-1} P_{x y}^{c}+2 A_{x x}\right) \\
P_{z z}^{k}=\frac{n}{2}(1+2 \alpha)^{-1}\left(1-\frac{4}{3} a n^{-1} P_{x y}^{c}-4 A_{x x}\right)
\end{gathered}
$$

$$
\begin{aligned}
P_{x y}^{k}= & -a \frac{n}{2}(1+2 \alpha)^{-2}\left[1-\frac{4}{3} a n^{-1} P_{x y}^{c}+2 A_{x x}\right. \\
& \left.-2 a^{-1}(1+2 \alpha) A_{x y}\right] .
\end{aligned}
$$

The tensor $A$ occurring in these equations is given by Eq. (3.12), which for uniform shear flow reduces to

$$
\begin{aligned}
\mathrm{A}= & \left.(m \beta)^{-1 / 2} n \sigma^{2} \chi_{e} \int d \hat{\boldsymbol{\sigma}}\left(\hat{\boldsymbol{\sigma}} \hat{\boldsymbol{\sigma}}-\frac{1}{3}\right]\right) \\
& \times \bar{a} \hat{\sigma}_{x} \hat{\sigma}_{y}\left[\frac{2}{\pi^{1 / 2}} \bar{a} \hat{\sigma}_{x} \hat{\sigma}_{y} e^{-\bar{a}^{2} \hat{\sigma}_{x}^{2} \hat{\sigma}_{y}^{2}}\right. \\
& \left.-\left(1+2 \bar{a}^{2} \hat{\sigma}_{x}^{2} \hat{\sigma}_{y}^{2}\right) \operatorname{erfc}\left(\bar{a} \hat{\sigma}_{x} \hat{\sigma}_{y}\right)\right],
\end{aligned}
$$

where $\bar{a} \equiv \frac{1}{2} a \sigma(m \beta)^{1 / 2}$. This gives directly the results (5.2) and (5.3).

For small shear rates the above results give $A=a \mathrm{~A}^{(1)}$ $+a^{2} \mathrm{~A}^{(2)}+a^{3} \mathrm{~A}^{(3)}+\cdots$, where the nonzero elements are $A_{x y}^{(1)}=-(2 \pi / 15) n^{*} \chi_{e}, \quad A_{x y}^{(3)}=-\left(256 \pi^{2} / 2625\right)\left(n^{*} \chi_{e}\right)^{3}$, and $A_{x x}^{(2)}=(128 \pi / 1575)\left(n^{*} \chi_{e}\right)^{2}$. Also, the condition for stationarity, Eq. (5.9), gives $\alpha(a) \rightarrow a^{2} \alpha^{(2)}+\cdots$, with $\alpha^{(2)}$ $=\frac{1}{3}\left(1-2 n^{-1} P_{x y}^{c(1)}-2 A_{x y}^{(1)}\right)$. Use of these results in Eqs. (C.2) $-(\mathrm{C} 5)$ gives

$$
\begin{aligned}
P_{x x}^{k}= & \frac{n}{2}\left[1+\frac{4}{3}\left(1+\frac{4 \pi}{15} n^{*} \chi_{e}+\frac{64 \pi}{525}\left(n^{*} \chi_{e}\right)^{2}\right) a^{2}+\ldots\right] \\
P_{y y}^{k}= & \frac{n}{2}\left[1-\frac{2}{3}\left(1+\frac{4 \pi}{15} n^{*} \chi_{e}-\frac{128 \pi}{525}\left(n^{*} \chi_{e}\right)^{2}\right) a^{2}+\ldots\right], \\
P_{z z}^{k}= & \frac{n}{2}\left[1-\frac{2}{3}\left(1+\frac{4 \pi}{15} n^{*} \chi_{e}+\frac{256 \pi}{525}\left(n^{*} \chi_{e}\right)^{2}\right) a^{2}+\cdots\right], \\
P_{x y}^{k}= & -\frac{n}{2} a\left\{1+\frac{4 \pi}{15} n^{*} \chi_{e}-\frac{4}{3}\left(\left(1-n^{-1} P_{x y}^{c(1)}+\frac{2 \pi}{15} n^{*} \chi_{e}\right)\right.\right. \\
& \left.\times\left(1+\frac{4 \pi}{15} n^{*} \chi_{e}\right)-\frac{64 \pi}{525}\left(n^{*} \chi_{e}\right)^{2}\left(1+\frac{6 \pi}{5}\left(n^{*} \chi_{e}\right)\right)\right] a^{2} \\
& +\ldots\} .
\end{aligned}
$$

Consider next the collisional transfer contribution to the momentum flux. Equation (2.15) becomes

$$
\begin{aligned}
\mathrm{P}^{c}= & \frac{m}{2} \sigma^{3} \chi_{e} \int d \hat{\boldsymbol{\sigma}} \hat{\boldsymbol{\sigma}} \hat{\boldsymbol{\sigma}} \int d \mathbf{V}_{1} \int d \mathbf{V}_{2} \Theta(\hat{\boldsymbol{\sigma}} \cdot \mathbf{g})(\hat{\boldsymbol{\sigma}} \cdot \mathbf{g})^{2} f\left(\mathbf{V}_{1}\right. \\
& +\mathrm{a} \cdot \boldsymbol{\sigma}) f\left(\mathbf{V}_{2}\right)
\end{aligned}
$$

The small shear rate dependence is obtained by the expansion (5.11) and 


$$
\begin{aligned}
f(\mathbf{V}+\mathbf{a} \cdot \boldsymbol{\sigma})= & f(\mathbf{V})+a \sigma \hat{\sigma}_{y} \frac{\partial}{\partial V_{x}} f(\mathbf{V})+\frac{a^{2}}{2} \sigma^{2} \hat{\sigma}_{y}^{2} \frac{\partial^{2}}{\partial V_{x}{ }^{2}} f(\mathbf{V}) \\
& +\frac{a^{3}}{6} \sigma^{3} \hat{\sigma}_{y}^{3} \frac{\partial^{3}}{\partial V_{x}{ }^{3}} f(\mathbf{V})+\cdots,
\end{aligned}
$$

to get

$$
\begin{aligned}
& P_{x y}^{c(1)}=\sigma^{3} \chi_{e} \int d \hat{\boldsymbol{\sigma}} \hat{\sigma}_{x} \hat{\sigma}_{y} \int d \mathbf{V}_{1} \int d \mathbf{V}_{2} \Theta(\hat{\boldsymbol{\sigma}} \cdot \mathbf{g})(\hat{\boldsymbol{\sigma}} \cdot \mathbf{g})^{2} \\
& \times\left[f^{(1)}\left(\mathbf{V}_{1}\right)+\frac{\sigma}{2} \hat{\sigma}_{y} \frac{\partial}{\partial V_{1 x}} f_{\lambda}\left(\mathbf{V}_{\mathbf{1}}\right)\right] f_{\lambda}\left(\mathbf{V}_{2}\right), \\
& P_{x x}^{c(2)}=\sigma^{3} \chi_{e} \int d \hat{\boldsymbol{\sigma}} \hat{\sigma}_{x}^{2} \int d \mathbf{V}_{1} \int d \mathbf{V}_{2} \Theta(\hat{\boldsymbol{\sigma}} \cdot \mathbf{g})(\hat{\boldsymbol{\sigma}} \cdot \mathbf{g})^{2} \\
& \times\left\{f^{(2)}\left(\mathbf{V}_{1}\right) f_{\ell}\left(\mathbf{V}_{2}\right)+\frac{1}{2} f^{(1)}\left(\mathbf{V}_{1}\right) f^{(1)}\left(\mathbf{V}_{2}\right)\right. \\
& +\frac{\sigma}{2} \hat{\sigma}_{y} \frac{\partial}{\partial V_{1 x}}\left[f^{(1)}\left(\mathbf{V}_{1}\right) f_{\curlywedge}\left(\mathbf{V}_{2}\right)+f_{\curlywedge}\left(\mathbf{V}_{1}\right) f^{(1)}\left(\mathbf{V}_{2}\right)\right] \\
& \left.+\frac{\sigma^{2}}{4} \hat{\sigma}_{y}^{2} \frac{\partial^{2}}{\partial V_{1 x}^{2}} f_{\ell}\left(\mathbf{V}_{1}\right) f_{\ell}\left(\mathbf{V}_{2}\right)\right\} \\
& P_{x y}^{c(3)}=\sigma^{3} \chi_{e} \int d \hat{\boldsymbol{\sigma}} \hat{\sigma}_{x} \hat{\sigma}_{y} \int d \mathbf{V}_{1} \int d \mathbf{V}_{2} \Theta(\hat{\boldsymbol{\sigma}} \cdot \mathbf{g})(\hat{\boldsymbol{\sigma}} \cdot \mathbf{g})^{2} \\
& \times\left\{f^{(3)}\left(\mathbf{V}_{1}\right) f,\left(\mathbf{V}_{2}\right)+f^{(2)}\left(\mathbf{V}_{1}\right) f^{(1)}\left(\mathbf{V}_{2}\right)+\frac{\sigma}{2} \hat{\sigma}_{y} \frac{\partial}{\partial V_{1 x}}\right. \\
& \times\left[f^{(2)}\left(\mathbf{V}_{1}\right) f_{\ell}\left(\mathbf{V}_{2}\right)+f^{(1)}\left(\mathbf{V}_{1}\right) f^{(1)}\left(\mathbf{V}_{2}\right)+f,\left(\mathbf{V}_{1}\right) f^{(2)}\right. \\
& \left.\times\left(\mathbf{V}_{2}\right)\right]+\frac{\sigma^{2}}{4} \hat{\sigma}_{y}^{2} \frac{\partial^{2}}{\partial V_{1 x}{ }^{2}}\left[f^{(1)}\left(\mathbf{V}_{1}\right) f_{\ell}\left(\mathbf{V}_{2}\right)+f_{\curlywedge}\left(\mathbf{V}_{1}\right) f^{(1)}\right. \\
& \left.\left.\times\left(\mathbf{V}_{2}\right)\right]+\frac{\sigma^{3}}{12} \hat{\sigma}_{y}^{3} \frac{\partial^{3}}{\partial V_{1 x}{ }^{3}} f_{\ell}\left(\mathbf{V}_{1}\right) f_{\ell}\left(\mathbf{V}_{2}\right)\right\} \text {. }
\end{aligned}
$$

The expressions for the elements $P_{y y}^{c(2)}$ and $P_{z z}^{c(2)}$ can be obtained from Eq. (C14) by replacing $\hat{\sigma}_{x}^{2}$ by $\hat{\sigma}_{y}^{2}$ and $\hat{\sigma}_{z}^{2}$, respectively. The integrations over $\hat{\boldsymbol{\sigma}}$ in Eqs. (C13)-(C15) can be performed using the identities

$$
\begin{gathered}
\int d \hat{\boldsymbol{\sigma}} \Theta(\hat{\boldsymbol{\sigma}} \cdot \mathbf{g})(\hat{\boldsymbol{\sigma}} \cdot \mathbf{g})^{2} \hat{\sigma}_{i} \hat{\sigma}_{j}=\frac{2 \pi}{15}\left(2 g_{i} g_{j}+g^{2} \delta_{i j}\right), \\
\int d \hat{\boldsymbol{\sigma}} \Theta(\hat{\boldsymbol{\sigma}} \cdot \mathbf{g})(\hat{\boldsymbol{\sigma}} \cdot \mathbf{g})^{2} \hat{\sigma}_{i} \hat{\sigma}_{j} \hat{\sigma}_{k} \\
=\frac{\pi}{12 g}\left[g_{i} g_{j} g_{k}+g^{2}\left(g_{i} \delta_{j k}+g_{j} \delta_{i k}+g_{k} \delta_{i j}\right)\right],
\end{gathered}
$$

$$
\begin{aligned}
& \int d \hat{\boldsymbol{\sigma}} \Theta(\hat{\boldsymbol{\sigma}} \cdot \mathbf{g})(\hat{\boldsymbol{\sigma}} \cdot \mathbf{g})^{2} \hat{\boldsymbol{\sigma}}_{i} \hat{\sigma}_{j} \hat{\sigma}_{k} \hat{\sigma}_{l}=\frac{2 \pi}{105}\left[2 \left(g_{i} g_{j} \delta_{k l}+g_{i} g_{k} \delta_{j l}\right.\right. \\
& \left.\quad+g_{i} g_{l} \delta_{j k}+g_{j} g_{k} \delta_{i l}+g_{j} g_{l} \delta_{i k}+g_{k} g_{l} \delta_{i j}\right)+g^{2}\left(\delta_{i j} \delta_{k l}\right. \\
& \left.\left.\quad+\delta_{i k} \delta_{j l}+\delta_{i l} \delta_{j k}\right)\right],
\end{aligned}
$$

$$
\int d \hat{\boldsymbol{\sigma}} \Theta(\hat{\boldsymbol{\sigma}} \cdot \mathbf{g})(\hat{\boldsymbol{\sigma}} \cdot \mathbf{g})^{2} \hat{\boldsymbol{\sigma}}_{x} \hat{\sigma}_{y}^{4}=\frac{\pi}{96 g^{3}} g_{x}\left(3 g^{4}+6 g^{2} g_{y}^{2}-g_{y}^{4}\right) .
$$

So far, Eqs. (C13) $-(\mathrm{C} 15)$ are also valid for the RET. The explicit forms for $f^{(1)}, f^{(2)}$, and $f^{(3)}$ follow from the formal solution to our kinetic model (5.7),

$$
\begin{aligned}
f^{(1)}(\mathbf{V})= & -2\left(1-2 A_{x y}^{(1)}\right) V_{x} V_{y} f_{\curlywedge}(\mathbf{V}) \\
f^{(2)}(\mathbf{V})= & {\left[\left(1-2 A_{x y}^{(1)}\right)\left(1-\frac{2}{3} V^{2}-2 V_{y}^{2}+4 V_{x}^{2} V_{y}^{2}\right)\right.} \\
+ & \left.2 A_{x x}^{(2)}\left(V^{2}-3 V_{z}^{2}\right)\right] f_{\ell}(\mathbf{V}), \\
f^{(3)}(\mathbf{V})= & 4 V_{x} V_{y}\left\{( 1 - 2 A _ { x y } ^ { ( 1 ) } ) \left[3 V_{y}^{2}-2 V_{x}^{2} V_{y}^{2}\right.\right. \\
& \left.+\frac{2}{3}\left(1-n^{-1} P_{x y}^{c(1)}-A_{x y}^{(1)}\right)\left(V^{2}-\frac{5}{2}\right)\right] \\
& \left.+A_{x y}^{(3)}-A_{x x}^{(2)}\left(V^{2}-3 V_{z}^{2}-1\right)\right\} f_{\ell}(\mathbf{V}) .
\end{aligned}
$$

With these results the velocity integrals of Eqs. (C13)-(C15) can be carried out by introducing the variables $\mathbf{G}=\frac{1}{2}\left(\mathbf{V}_{1}\right.$ $+\mathbf{V}_{2}$ ) and $\mathbf{g}=\mathbf{V}_{1}-\mathbf{V}_{2}$ to get

$$
\begin{aligned}
P_{x y}^{c(1)}= & -\frac{n}{2} \frac{4 \pi}{15} n^{*} \chi_{e}\left(1-2 A_{x y}^{(1)}+\frac{16}{5} n^{*} \chi_{e}\right), \\
P_{x x}^{c(2)}= & \frac{n}{2} \frac{16 \pi}{45} n^{*} \chi_{e}\left[\left(1+\frac{36}{35} n^{*} \chi_{e}\right)\left(1-2 A_{x y}^{(1)}\right)\right. \\
& \left.+\frac{144 \pi}{175}\left(n^{*} \chi_{e}\right)^{2}+\frac{3}{2} A_{x x}^{(2)}\right],
\end{aligned}
$$

$$
\begin{aligned}
P_{y y}^{c(2)}= & \frac{n}{2} \frac{16 \pi}{45} n^{*} \chi_{e}\left[-\left(\frac{1}{2}-\frac{36}{35} n^{*} \chi_{e}\right)\left(1-2 A_{x y}^{(1)}\right)\right. \\
& \left.+\frac{144 \pi}{175}\left(n^{*} \chi_{e}\right)^{2}+\frac{3}{2} A_{x x}^{(2)}\right],
\end{aligned}
$$

$$
\begin{aligned}
P_{z z}^{c(2)}= & \frac{n}{2} \frac{16 \pi}{45} n^{*} \chi_{e}\left[-\left(\frac{1}{2}-\frac{12}{35} n^{*} \chi_{e}\right)\left(1-2 A_{x y}^{(1)}\right)\right. \\
& \left.+\frac{48 \pi}{175}\left(n^{*} \chi_{e}\right)^{2}-3 A_{x x}^{(2)}\right],
\end{aligned}
$$




$$
\begin{aligned}
P_{x y}^{c(3)}= & \frac{n}{2} \frac{16 \pi}{45} n^{*} \chi_{e}\left\{\left[1-n^{-1} P_{x y}^{c(1)}-A_{x y}^{(1)}\left(1+\frac{6}{35} n^{*} \chi_{e}\right)\right.\right. \\
& \left.+\frac{1}{35} n^{*} \chi_{e}\right]\left(1-2 A_{x y}^{(1)}\right)-\frac{256 \pi}{875}\left(n^{*} \chi_{e}\right)^{3} \\
& \left.-\frac{3}{2} A_{x x}^{(2)}\left(1+\frac{32}{35} n^{*} \chi_{e}\right)+\frac{3}{2} A_{x y}^{(3)}\right\} .
\end{aligned}
$$

These small shear rate results for $\mathrm{P}^{k}$ and $\mathrm{P}^{c}$ allow calculation of the shear viscosity, viscometric functions, and shear dilatancy. For example, the kinetic parts are found to be

$$
\begin{aligned}
\eta^{k}(a)= & \frac{n}{2}\left(1+\frac{4 \pi}{15} n^{*} \chi_{e}\right)-\frac{n}{2}\left[\frac{4}{3}+\frac{32 \pi}{15} n^{*} \chi_{e}\right. \\
& +\frac{32 \pi}{45}\left(\frac{4}{7}+\frac{\pi}{5}\right)\left(n^{*} \chi_{e}\right)^{2}-\frac{128 \pi^{2}}{3375}\left(\frac{8}{7}-\frac{\pi}{3}\right) \\
& \left.\times\left(n^{*} \chi_{e}\right)^{3}\right] a^{2}+O\left(a^{4}\right),
\end{aligned}
$$

$$
\begin{aligned}
& \Psi_{1}^{k}(a)=-\frac{n}{2}\left(2+\frac{8 \pi}{15} n^{*} \chi_{e}\right)+O\left(a^{2}\right), \\
& \Psi_{2}^{k}(a)=-\frac{n}{2} \frac{256 \pi}{525}\left(n^{*} \chi_{e}\right)^{2}+O\left(a^{2}\right) .
\end{aligned}
$$

The collisional transfer contributions are calculated in a similar way, leading to the results quoted in Sec. V.

To study the case of asymptotically large values of the shear rate, the results (5.26) for the collisional transfer contributions to the pressure tensor can be compared to those for the kinetic parts. Using Eq. (5.27) we obtain from Eqs. (C2) $-(\mathrm{C} 5)$

$$
\begin{gathered}
P_{x x}^{k} \approx P_{y y}^{k} \approx 4 P_{z z}^{k} \approx \frac{2}{3} n, \\
P_{x y}^{k} \approx-\frac{3 \pi}{16} n .
\end{gathered}
$$

Comparison with Eq. (5.27) shows that even in the dilute regime $\left(n^{*} \ll 1\right)$ the collisional transfer part of the pressure tensor dominates over the kinetic part for asymptotically large shear rates. In particular, $\eta(a) \sim a$, which shows that for densities lower than $n^{*} \simeq 0.79$ there is a crossover from shear thinning to shear thickening at sufficiently large shear rates.
[1] G. Bird, Molecular Gas Dynamics and the Direct Simulation of Gas Flows (Clarendon Press, Oxford, 1994).

[2] For a recent review see J. W. Dufty, in Lectures on Thermodynamics and Statistical Mechanics, edited by M. López de Haro and C. Varea (World Scientific, New Jersey, 1990), pp. $166-181$.

[3] J. Gómez Ordóñez, J. J. Brey, and A. Santos, Phys. Rev. A 41, 810 (1990); J. M. Montanero, M. Alaoui, A. Santos, and V. Garzó, Phys. Rev. E 49, 367 (1994); J. M. Montanero, A. Santos, and V. Garzó, Phys. Fluids 8, 1981 (1996); J. Stat. Phys. 88, 1165 (1997).

[4] H. van Beijeren and M. H. Ernst, J. Stat. Phys. 21, 125 (1979).

[5] P. Résibois, J. Stat. Phys. 19, 593 (1978); J. Piasecki, ibid. 48, 1203 (1987).

[6] S. Yip, W. Alley, and B. Alder, J. Stat. Phys. 27, 201 (1982).

[7] The single exception we are aware of is the recent extension of Grad's moment method approximation to the RET by J. F. Lutsko, Phys. Rev. Lett. 78, 243 (1997).

[8] J. M. Montanero and A. Santos, Phys. Rev. E 54, 438 (1996); Phys. Fluids 9, 2057 (1997).

[9] J. W. Dufty, A. Santos, and J. J. Brey, Phys. Rev. Lett. 77, 1270 (1996).

[10] J. J. Brey, J. W. Dufty, and A. Santos, J. Stat. Phys. 87, 1051 (1997).

[11] J. W. Dufty, J. J. Brey, and A. Santos, Physica A 240, 212 (1997).

[12] C. Cercignani, Theory and Application of the Boltzmann Equation (Elsevier, New York, 1975).

[13] R. Evans, Adv. Phys. 28, 143 (1979).
[14] T. R. Kirkpatrick, S. P. Das, M. H. Ernst, and J. Piasecki, J. Chem. Phys. 92, 3768 (1990).

[15] J. W. Dufty, M. Lindenfeld, and G. Garland, Phys. Rev. A 24, 3212 (1981).

[16] G. Szamel and M. H. Ernst, Phys. Rev. B 48, 112 (1993); G. Szamel, J. Stat. Phys. 87, 1067 (1997).

[17] J. A. McLennan, Introduction to Nonequilibrium Statistical Mechanics (Prentice-Hall, Englewood Cliffs, NJ, 1989).

[18] J. H. Ferziger and H. G. Kaper, Mathematical Theory of Transport Processes in Gases (North-Holland, Amsterdam, 1972).

[19] N. F. Carnahan and K. E. Starling, J. Chem. Phys. 51, 635 (1969).

[20] Nonlinear Fluid Behavior, edited by H. J. M. Hanley (NorthHolland, Amsterdam, 1983).

[21] A. W. Lees and S. F. Edwards, J. Phys. C 5, 1921 (1972).

[22] J. W. Dufty, J. J. Brey, and A. Santos, in Molecular-Dynamics Simulation of Statistical-Mechanical Systems, edited by G. Ciccotti and W. G. Hoover (North-Holland, Amsterdam, 1986), pp. 294-303.

[23] J. W. Dufty, A. Santos, J. J. Brey, and R. F. Rodríguez, Phys. Rev. A 33, 459 (1986).

[24] B. J. Alder, W. G. Hoover, and D. A. Young, J. Chem. Phys. 49, 3688 (1968).

[25] J. M. Montanero and A. Santos, Physica A 240, 229 (1997).

[26] P. G. De Gennes, Rev. Mod. Phys. 57, 827 (1985); J. Koplik, J. Banavar, and J. Willemsen, in Microscopic Simulations of Complex Flows, edited by M. Mareschal (Plenum Press, New York, 1989).

[27] J. J. Brey, M. J. Ruiz-Montero, and F. Moreno, Phys. Rev. E 55, 2846 (1997). 